\title{
On the Standardization of Approximate Entropy: Multidimensional Approximate Entropy Index Evaluated on Short-Term HRV Time Series
}

\author{
Juan Bolea $\mathbb{D}^{1,2}$ Raquel Bailón, ${ }^{1,2}$ and Esther Pueyo ${ }^{1,2}$ \\ ${ }^{1}$ BSICoS Group, Aragón Institute of Engineering Research (I3A), IIS Aragón, Universidad de Zaragoza, Zaragoza, Spain \\ ${ }^{2}$ Centro de Investigación Biomédica en Red: Bioingeniería, Biomateriales y Nanomedicina (CIBER), Madrid, Spain
}

Correspondence should be addressed to Juan Bolea; jbolea@unizar.es

Received 30 April 2018; Revised 22 August 2018; Accepted 29 October 2018; Published 5 November 2018

Guest Editor: Daniel Novák

Copyright (C) 2018 Juan Bolea et al. This is an open access article distributed under the Creative Commons Attribution License, which permits unrestricted use, distribution, and reproduction in any medium, provided the original work is properly cited.

\begin{abstract}
Background. Nonlinear heart rate variability (HRV) indices have extended the description of autonomic nervous system (ANS) regulation of the heart. One of those indices is approximate entropy, ApEn, which has become a commonly used measure of the irregularity of a time series. To calculate ApEn, a priori definition of parameters like the threshold on similarity and the embedding dimension is required, which has been shown to be critical for interpretation of the results. Thus, searching for a parameter-free $A p E n$-based index could be advantageous for standardizing the use and interpretation of this widely applied entropy measurement. Methods. A novel entropy index called multidimensional approximate entropy, $M A p E n_{\max }$, is proposed based on summing the contribution of maximum approximate entropies over a wide range of embedding dimensions while selecting the similarity threshold leading to maximum ApEn value in each dimension. Synthetic RR interval time series with varying levels of stochasticity, generated by both $\operatorname{MIX}(P)$ processes and white/pink noise, were used to validate the properties of the proposed index. Aging and congestive heart failure (CHF) were characterized from RR interval time series of available databases. Results. In synthetic time series, $M A p E n_{\max }$ values were proportional to the level of randomness; i.e., $M A p E n_{\max }$ increased for higher values of $P$ in generated $\operatorname{MIX}(P)$ processes and was larger for white than for pink noise. This result was a consequence of all maximum approximate entropy values being increased for higher levels of randomness in all considered embedding dimensions. This is in contrast to the results obtained for approximate entropies computed with a fixed similarity threshold, which presented inconsistent results for different embedding dimensions. Evaluation of the proposed index on available databases revealed that aging was associated with a notable reduction in $M A p E n_{\max }$ values. On the other hand, $M A p E n_{\max }$ evaluated during the night period was considerably larger in CHF patients than in healthy subjects. Conclusion. A novel parameter-free multidimensional approximate entropy index, $M A p E n_{\text {max }}$, is proposed and tested over synthetic data to confirm its capacity to represent a range of randomness levels in HRV time series. MApEn $n_{\max }$ values are reduced in elderly patients, which may correspond to the reported loss of ANS adaptability in this population segment. Increased $M A p E n_{\max }$ values measured in CHF patients versus healthy subjects during the night period point to greater irregularity of heart rate dynamics caused by the disease.
\end{abstract}

\section{Introduction}

Approximate entropy (ApEn) was introduced by Pincus and coworkers [1] in 1991 as an entropic measurement to quantify the regularity of medical data. Ten years later, Richmann and Moorman [2] introduced sample entropy (SampEn), a variation of $A p E n$ reducing the bias of considering selfcomparisons and being more independent of data length. Both approaches have been widely used to characterize medical disorders or discriminate between healthy and pathological conditions [3-9]. However, these entropy measures are by definition dependent on two predefined parameter values, namely: $m$, the embedding dimension (i.e., the length of reconstructed vectors), and $r$, the tolerance threshold (i.e., similarity value for comparing reconstructed vectors). These parameters have been assigned diverse values in published heart rate variability (HRV) studies (e.g., $m$ $=1$ or $2 ; r=0.1$ to 0.25 times the standard deviation of 
the time series) [9-19]. Thus, one main drawback of these entropy indices lies in the comparison between studies, since diverse values of a priori parameters can lead to different physiological interpretations.

Entropy-based methods, and in particular SampEn-based ones, have received great attention in the last ten to fifteen years. Alternatives have been proposed to make this index independent of a priori parameter definition, thus facilitating the standardization of its application. In this regard, multiscale entropy (MSE) and refined MSE approaches were introduced to take into account complexity properties at different scales by applying the coarse-graining technique $[15,20-22]$. Each scale was characterized by a new time series derived from the original one by averaging and decimation [15]. However, they still required the predefined parameters $m$ and $r$. Alternatives, such as the use of a density function instead of a tolerance value, was proposed to improve estimation of Renyi entropy, generalizing the Shannon entropy [23, 24]. Other entropy-based approaches have been proposed to avoid the need for predefining specific parameters. In this regard, permutation entropy of a given order was calculated from the relative frequencies of all permutations of that order of the analyzed time series [25]. Dispersion entropy was suggested as a more reliable method than sample or permutation entropies. However, the need for defining the tolerance threshold $r$ is not strictly avoided, but this is replaced with the definition of a number of classes [26]. Rankbased methods were investigated to quantify the amount of shuffling by sorting the ranks of the mutual distances between pairs of $m$-long vectors [27]. Although this approach avoids predefinition of the threshold value, the embedding dimension requires a priori definition. On the other hand, the cumulative histogram was proposed to overcome the limitation of defining a tolerance $r$ value in the computation of sample entropy, yielding the approximate entropy profile [28]. An approach to measure bubble entropy, which was shown to be independent of the embedding dimension $m$ for sufficiently large values of this parameter, was introduced in [29]. Several of the above described approaches showed good performance to characterize pathological versus healthy conditions and led to a reduction in the associated computational load. Nevertheless, they still presented dependence on some parameters, like the embedding dimension or the tolerance value.

Although SampEn was introduced as an improvement of $A p E n$ entropy, advances towards the standardization of ApEn have been investigated as well. Different approaches have been proposed to identify the values of the embedding dimension $m$ or the threshold $r$ used for the characterization of nonlinear dynamics in chaotic time series [30-35]. With regard to the embedding dimension, the false nearest neighbor method was used to search for the lowest embedding dimension $m$ that allows phase-space reconstruction. Very different values of $m$ were reported for different models and experimental data [30]. The lowest values of $m$ were found to be 2, 3, and 6 for Hénon, Lorenz, and Mackey-Glass time series, respectively [31]. With regard to the tolerance threshold $r$, some studies focused on searching for the value, denoted by $r_{\max }(m)$, that maximizes ApEn $[32,33,36]$. The corresponding $A p E n$-based index, maximum $A p E n$, was used to show that white noise series was more irregular than crosschirp signal [37]. In addition, this index was reported to better enhance HRV and blood pressure variability differences between supine and upright position with respect to the use of fixed $r$ values, typically $0.1-0.25$ times the standard deviation [34]. ApEn profile, based on cumulative histogram computations, was proposed to avoid the shortcomings of a priori definition of the tolerance threshold [38]. HRV indices derived from such ApEn profiles were shown to better separate young versus old individuals as compared to classical ApEn approach. To reduce the influence of the threshold $r$ on the previous method, an adaptive cumulative histogram method was proposed [39]. Total and average cross-approximate entropies, as well as the standard deviation of cross-approximate entropy, distinguished financial time series successfully. It should be noted that although $r$ is generally referred to as a fixed value, the parameter that is fixed is the factor multiplying the standard deviation of the analyzed time series, rather than the threshold $r$ itself. In the literature, identification of the values of $r$ and $m$ used to characterize different time series is still unclear, as highlighted by the highly diverse published values $[34,35]$.

In this study, we aimed to introduce an ApEn-based index that avoids the need for a priori parameter definition and, thus, allows standardization of entropy-based measurements. In particular, $M A p E n_{\max }$ is proposed based on summation of maximum ApEn values over different embedding dimensions for each given HRV time series. This multidimensional index does not require the use of the coarse-graining technique and overcomes the variations in data length for different time scales. Furthermore, its computation provides entropic estimation without the need for ad hoc selection of input parameter values. The ability of the proposed index $M A p E n_{\max }$ to represent different degrees of randomness in time series is first tested in synthetically generated signals and subsequently used to characterize HRV changes induced by aging and by congestive heart failure (CHF).

\section{Materials and Methods}

2.1. Materials. MIX processes: a family of processes combining deterministic and stochastic behavior were studied. The degree of stochasticity was controlled by parameter $P$. $\operatorname{MIX}(P)$ generated a sine for $P=0$ (pure deterministic) and became more random as $P$ increased up to $1(P=1$, pure stochastic):

$$
\operatorname{MIX}(P)_{j}=\left(1-Z_{j}\right) X_{j}+Z_{j} Y_{j},
$$

where $X_{j}=\sqrt{2} \sin (2 j \pi / 12), Y_{j} \sim$ are i.i.d. uniform random variables on $[-\sqrt{3} \sqrt{3}]$, and $Z_{j} \sim$ are i.i.d. random variables, with $Z_{j}=1$ having probability $P$ and $Z_{j}=0$ probability 1$P$ [40]. For each $P$ value in $\{0,0.25,0.5,0.75,1\}, 30$ processes were generated, each containing 300 samples.

Noise time series: (i) White and pink noise $(1 / f)$ time series with zero mean and unit variance were studied. A total of 30 white and pink noise processes, respectively, were generated, each containing 300 samples. (ii) Bandpass 
filtered noise derived from white noise time series filtered in two frequency passbands, representing the low (LF) and high (HF) frequency components, with variable bandwidths. Specifically, three types of bandpass filters with the following passband limits were considered: $\mathrm{BW} 1(\mathrm{LF}=0.09-0.13 \mathrm{~Hz}$ and $\mathrm{HF}=0.22-0.28 \mathrm{~Hz})$; BW2 $(\mathrm{LF}=0.07-0.15 \mathrm{~Hz}$ and $\mathrm{HF}=0.19$ $0.31 \mathrm{~Hz})$; and BW3 $(\mathrm{LF}=0.05-0.17 \mathrm{~Hz}$ and $\mathrm{HF}=0.16-0.34 \mathrm{~Hz})$. In all cases, the power of LF and HF spectral components, $P_{L F}(0.04-0.15 \mathrm{~Hz})$ and $P_{H F}(0.15-0.4 \mathrm{~Hz})$, remained invariant. A total of 30 filtered white noise time series per type were generated.

2.1.1. Fantasia Database. Heartbeat locations from continuous electrocardiographic (ECG) signals sampled at $250 \mathrm{~Hz}$ were available from twenty young (21-34 y.o., mean 25.9) and 20 elderly rigorously screened healthy subjects (68-85 y.o., mean 74.5) undergoing 120 minutes of supine resting while watching the movie Fantasia (Disney, 1940) to help maintain wakefulness. Further database information is available in [41]. Recordings can be downloaded from http://www.physionet.org [42]. The whole 2-hour recordings were analyzed.

\subsubsection{Congestive Heart Failure and Normal Sinus Rhythm} Databases. For the CHF cohort, heartbeat locations from 44 CHF patients, aged $55.5 \pm 11.4$ y.o., of the Congestive Heart Failure RR Interval Database and the BIDMC Congestive Heart Failure Database (including patients in all NYHA classes) were considered.

For the healthy population, heartbeat locations from 72 healthy subjects, aged $54.6 \pm 16$ y.o., of the MIT-BIH Normal Sinus Rhythm Database and the Normal Sinus Rhythm RR Interval Database were considered.

These databases were available from http://www.physionet.org [42]. The analyzed heartbeat locations of all databases were obtained from an automatic ECG annotator and were subsequently manually reviewed and corrected by experts among Physionet collaborators. In this study, those heartbeat locations were corrected for ectopic beats based on instantaneous heart rate variation [43]. Subsequently, the time intervals between consecutive heartbeats were used to define the RR interval time series. Each RR time series was divided into 300 -sample segments, with $50 \%$ overlapping, and interpolated at $2 \mathrm{~Hz}$ to attenuate the effect of heart rate mean as sampling rate [44]. A 3-hour night period centered on the 300-sample segment with minimum heart rate mean $(H R M)$ was selected in all cases to minimize the influence caused by potentially different daily activities.

Estimation of linear and nonlinear HRV indices was performed on each segment. Subsequently, the median value of each index over those segments was computed as the representative value for each of the analyzed time series.

\subsection{Methods}

2.2.1. Approximate Entropy. Let $x(n), n=1, \ldots, N$, be the time series of interest, which is normalized by its standard deviation and $\boldsymbol{y}_{i}^{m}$ the $i$ th reconstructed vector of embedding dimension $m$.

$$
\boldsymbol{y}_{i}^{m}=[x(i), x(i+1), x(i+2), \ldots, x(i+(m-1))]^{T}
$$

The amount of reconstructed vectors was $N_{m}=N-(m-1)$ for each embedding dimension $m$. The distance, computed by the $L_{\infty}$ norm, between each pair of reconstructed vectors, $\boldsymbol{y}_{i}^{m}, \boldsymbol{y}_{j}^{m}$, was denoted by $d_{i, j}^{m}$. Each distance was compared with a threshold $r$ to compute the number of reconstructed vectors that lie within a hyperspace centered in the reconstructed vector of reference:

$$
C_{i}^{m}(r)=\frac{1}{N_{m}} \sum_{j=1}^{N_{m}} H\left(r-d_{i, j}^{m}\right),
$$

where $C_{i}^{m}$ is the correlation sum and $H$ is the Heaviside function.

This procedure was repeated with all reconstructed vectors and the probability of a pattern of length $m$ appearing along the time series was denoted by the following.

$$
\begin{aligned}
\phi^{m}(r) & =\frac{1}{N_{m}} \sum_{i=1}^{N_{m}} \log \left(C_{i}^{m}(r)\right) \\
\operatorname{ApEn}(m, r) & =\phi^{m}(r)-\phi^{m+1}(r)
\end{aligned}
$$

In this study, $m=2$ and $r=0.2$ were considered, leading to computation of $\operatorname{ApEn}(2,0.2)$.

2.2.2. Maximum Approximate Entropy. For each embedding dimension $m$, the threshold $r$ leading to maximum approximate entropy, denoted by $r_{\max }(m)$, was determined as in [45] and the maximum value $\operatorname{ApEn}\left(m, r_{\max }(m)\right)$ was calculated [36]. For the sake of comparison with $\operatorname{ApEn}(2,0.2), \operatorname{ApEn}(2$, $\left.r_{\max }(2)\right)$ was calculated.

2.2.3. Multidimensional Approximate Entropy. The multidimensional entropy index, $M A p E n(r)$, was introduced by summing up the approximate entropies for a range of embedding dimensions:

$$
\operatorname{MApEn}(r)=\sum_{m=1}^{m=m_{\max }} \operatorname{ApEn}(m, r)
$$

where $m_{\max }=15$. To additionally remove the dependence of the tolerance threshold, the value $r_{\max }(m)$ was identified for each embedding dimension $\mathrm{m}$, leading to the definition of $M A p E n_{\text {max }}$.

$$
M A p E n_{\max }=\sum_{m=1}^{m=m_{\max }} \operatorname{ApEn}\left(m, r_{\max }(m)\right)
$$

ApEn, SampEn, and correlation dimension $\mathrm{D}_{2}$ were computed using the methodology described in [45], considering a range for the tolerance threshold $r$ varying from 0.01 to 3 times the standard deviation of the time series with $a$ resolution of 0.01 . 


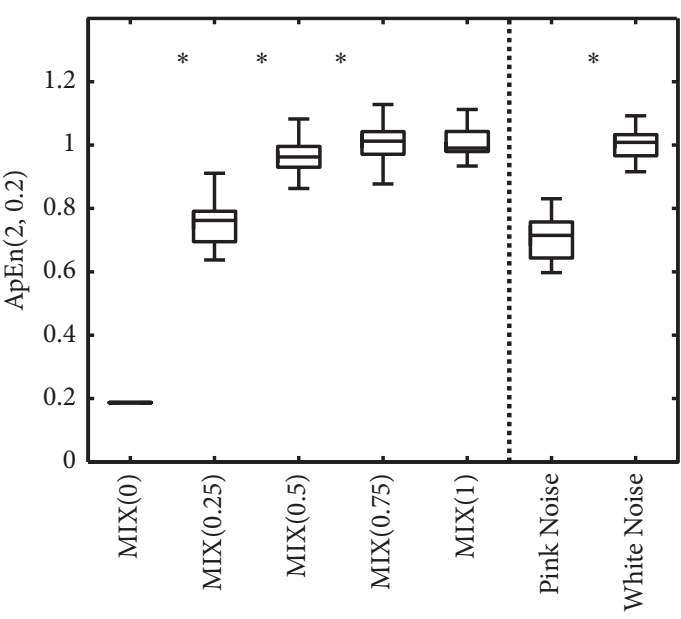

(a)

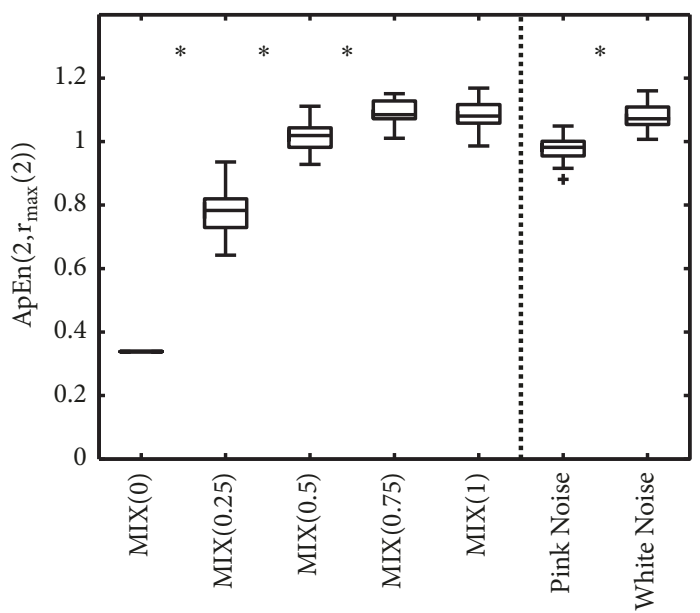

(c)

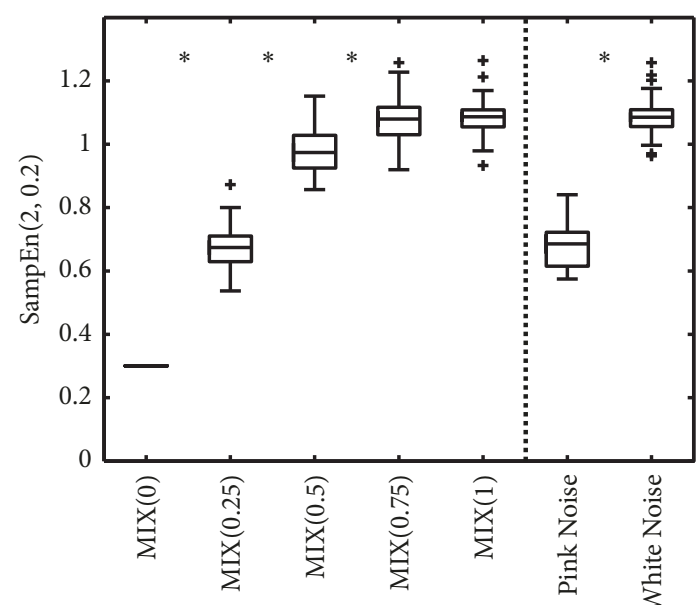

(b)

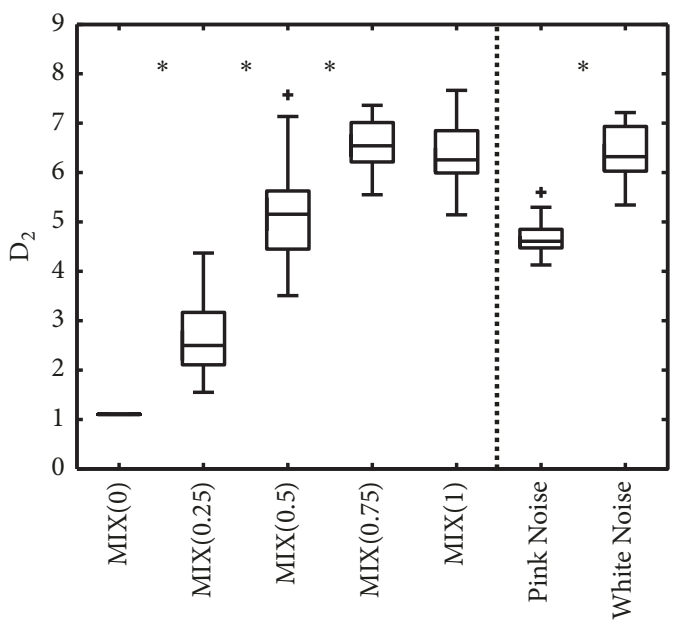

(d)

Figure 1: Nonlinear indices: (a) $A p E n(2,0.2)$; (b) $\operatorname{SampEn}(2,0.2)$; (c) $A p E n\left(2, r_{\max }(2)\right)$; and (d) $D_{2}$ evaluated over MIX(P) processes with varying stochastic levels and pink and white noise time series. $*$ indicates statistically significant differences by Mann-Whitney $U$ test $(p$ values $<0.05)$ when comparing $\operatorname{MIX}(P)$ processes or pink versus white noise time series. Data are shown as median and interquartile range over 30 realizations.

2.2.4. Time- and Frequency-Domain HRV Indices. Time- and frequency-domain indices were obtained from the raw RR time series and from the modulating signal of the integral pulse frequency modulation (IPFM) model, respectively, using 300 heartbeats as analysis windows.

$H R M$ and the square root of the mean squared differences of successive normal heartbeat intervals (RMSSD) were calculated.

Spectral analysis was performed on a modulating signal, $m(t)$, assumed to carry information about the autonomic nervous system (ANS) activity, which was estimated based on the IPFM model compensating for the influence of HRM [46]. Power in the low frequency $\left(P_{L F}, 0.04-0.15 \mathrm{~Hz}\right)$, related to sympathetic and parasympathetic activity, and high frequency bands $\left(P_{H F}, 0.15-0.4 \mathrm{~Hz}\right)$, as a parasympathetic modulation estimation, as well as normalized low frequency power $\left(P_{L F n}=P_{L F} /\left(P_{L F}+P_{H F}\right)\right)$ was estimated [47].
2.3. Statistical Analysis. The normality of data distributions was tested by the Kolmogorov-Smirnov test. Nonlinear as well as time- and frequency-domain HRV indices were compared between young and elderly subjects and between CHF patients and healthy subjects by t-test or Mann-Whitney $\mathrm{U}$ test depending on whether data distributions fulfilled the normality criterion or not. Only paired comparisons (rather than other multiple comparisons) were performed. Spearman correlation between the proposed index $M A p E n_{\max }$ and other HRV indices was computed.

\section{Results}

3.1. Synthetic Data. Figures 1 and 2 show the values of the analyzed nonlinear HRV indices computed over $\operatorname{MIX}(P)$ processes and pink and white noise time series. In the comparison of $\operatorname{MIX}(P)$ processes with randomness degree below $P=0.75$ ( 0 versus $0.25 ; 0.25$ versus 0.5 ; and 0.5 


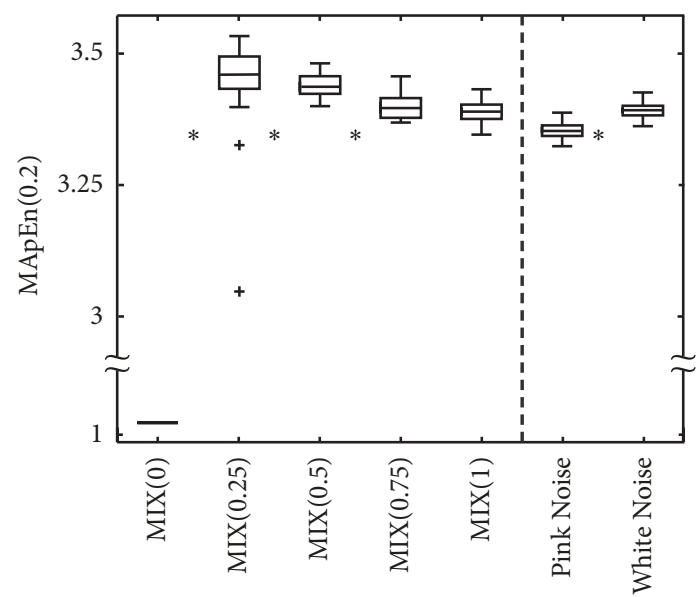

(a)

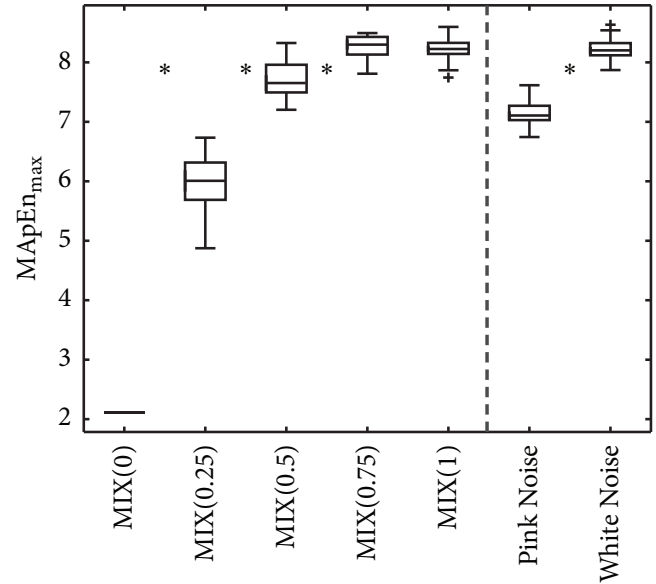

(b)

FIGURE 2: (a) $M A p E n(0.2)$ and (b) $M A p E n_{\text {max }}$ evaluated over $\operatorname{MIX}(P)$ processes with varying stochastic levels as well as white and pink noise. $(*)$ indicates statistically significant differences by Mann-Whitney $U$ test ( $p$-values $<0.05)$ when comparing MIX $(P)$ processes or pink versus white noise time series. Data are shown as median and interquartile range over 30 realizations.

versus 0.75 ) all nonlinear indices led to statistically significant differences. In the comparison between $\operatorname{MIX}(P)$ processes with $P$ above 0.75 ( 0.75 versus 1$)$ no statistically significant differences were found for any of the nonlinear indices. $A p E n(2,0.2)$ and $A p E n\left(2, r_{\max }(2)\right)$ as well as nonlinear index values were higher for greater levels of randomness until MIX(0.75); however above $P=0.75$ an increase in the level of randomness was not captured by ApEn or by any of the nonlinear indices. Regarding the separation of pink and white noise time series, all the analyzed indices were able to separate them. The range of $r_{\max }(2)$ values was found to lie in the interval 0.2-0.3 times the standard deviation of the time series, which is commonly used in the literature (see Table S1 in Supplementary Materials for additional information). The multidimensional index $\operatorname{MApEn}(r)$ was able to separate pink and white noise time series for all tested values of the tolerance threshold $r$ (results only shown for $r=0.2$, Figure 2(a)).

While statistically significant differences were found for both $M A p E n(0.2)$ and $M A p E n_{\text {max }}$ when comparing different stochastic processes, the former presented decreasing values as randomness increased, while the latter showed opposite results. To analyze these differences, the results of evaluating ApEn for two embedding dimensions, namely, $m=2$ and $m=$ 6 , are illustrated in Figure 3. The use of a fixed threshold did not always allow ApEn to characterize the randomness level of the $\operatorname{MIX}(P)$ processes. As an example, for $m=6$, the use of the fixed threshold $r=0.2$ led to $\operatorname{MIX}(0.25)$ having much larger complexity than MIX(0.5), MIX(0.75), and MIX(1). For $m=2$, however, $\operatorname{MIX}(0.25)$ presented the lowest complexity. When combining results from different scales, $\operatorname{MApEn}(0.2)$ showed lower values as the randomness level increased, thus not being able to reflect the degree of stochasticity in the time series (Figure 2(a)). However, the use of $A p E n\left(m, r_{\text {max }}(m)\right)$ when computing $M A p E n_{\text {max }}$ served to characterize $\operatorname{MIX}(P)$ processes according to their stochasticity level for any value of $m$, which then rendered $M A p E n_{\max }$ suitable to characterize stochasticity levels (Figure 2(b)).

In Figures 4(a) and 4(b) $A p E n(m, 0.2)$ and $A p E n(m$, $\left.r_{\text {max }}(m)\right)$ are shown for pink and white noise when the embedding dimension $m$ was varied from 1 to 15 . ApEn $(m$, 0.2 ) values were higher for white noise than for pink noise for low embedding dimension values, while presenting the opposite behavior for large embedding dimensions. The multidimensional index $M A p E n(0.2)$ resulted in higher values for white than for pink noise, reflecting the behavior found for the lowest embedding dimensions (Figure 2(a)). On the other hand, $\operatorname{ApEn}\left(m, r_{\max }(m)\right)$ was consistently larger for white than pink noise across all embedding dimensions and, consequently, differences between these noise time series were amplified when computing $M A p E n_{\max }$ (Figure 2(b)) with respect to $\operatorname{MApEn}(0.2)$ (Figure 2(a)).

As the statistical significance in the comparison of simulated data highly depends on the number of simulated processes (30 in the comparisons shown in Figure 2(b)), a study was performed to characterize such a dependence for the proposed index $M A p E n_{\max }$ when this is compared in all cases shown in Figure 2(b). Results are presented in Table 1 for a number of processes varying from 5 to 30 in 5 -process steps. As shown in the table, $p$-values decreased as the number of simulated processes increased. For a $p$ value of 0.05 , statistical significance was already achieved for a number of simulated processes as low as 5 in all comparisons indicated as significant in the results presented in Figure 2(b) corresponding to 30 simulated processes.

The median spectrum obtained from each type of bandpass filtered noise time series is illustrated in Figure 5(a). Time- and frequency-domain indices, as well as the proposed $M A p E n_{\text {max }}$ index, were estimated over these filtered noise time series (see Figure S4 in Supplementary Materials for time- and frequency-domain indices). Statistically significant differences when comparing the different filtered noise time 


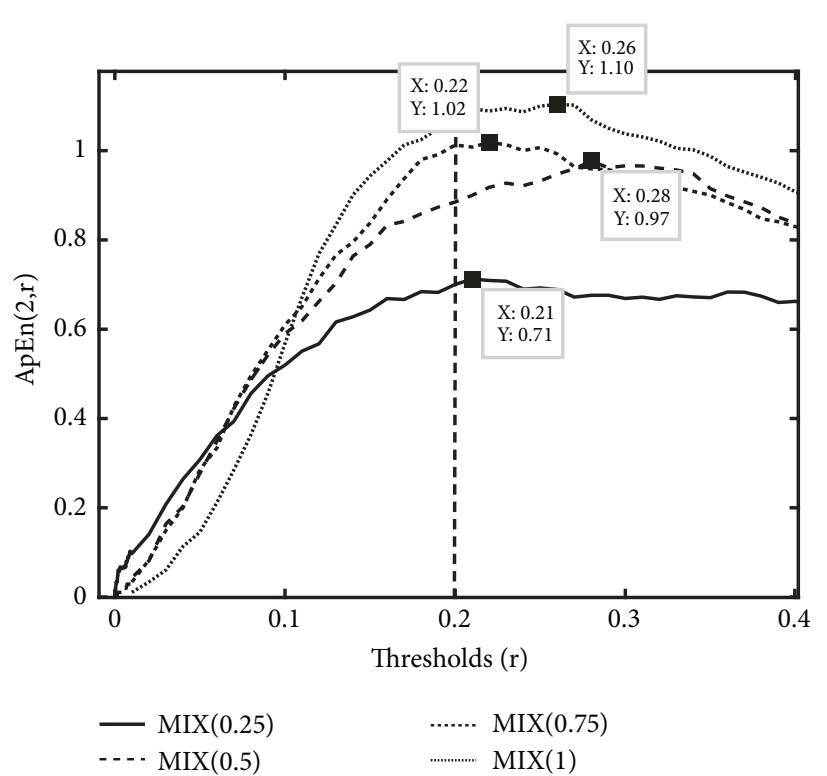

(a)

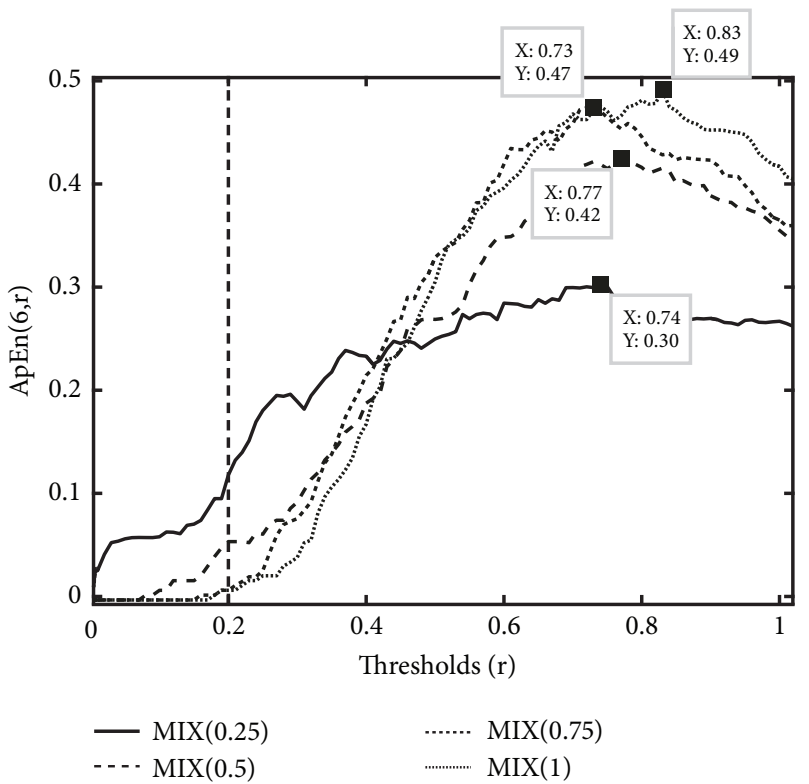

(b)

Figure 3: Approximate entropy values computed over $\operatorname{MIX}(P)$ processes considering a set of thresholds and evaluated for $m=2(\mathrm{a})$ and $m=6$ (b) for a particular stochastic realization. Dashed line indicates $r=0.2$ in both panels. ApEn(2, $\left.r_{\max }(2)\right), A p E n\left(6, r_{\max }(6)\right), r_{\max }(2)$, and $r_{\max }(6)$ values are displayed for each case.

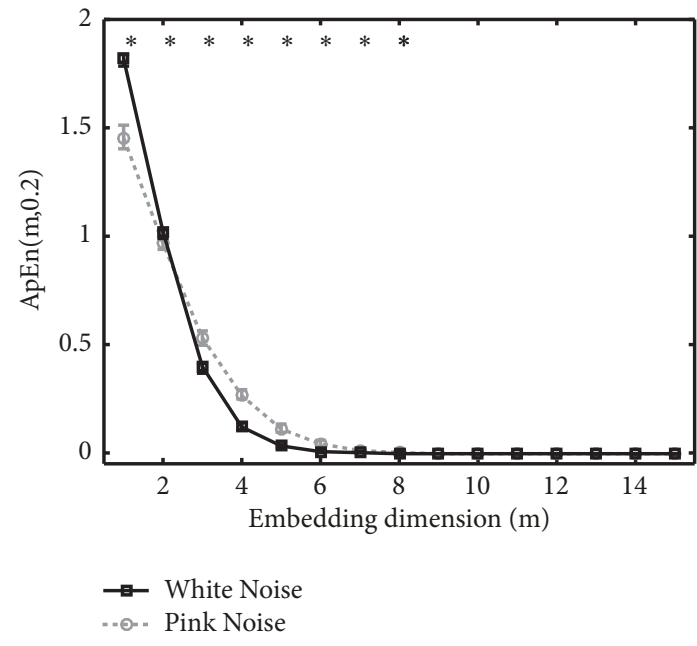

(a)

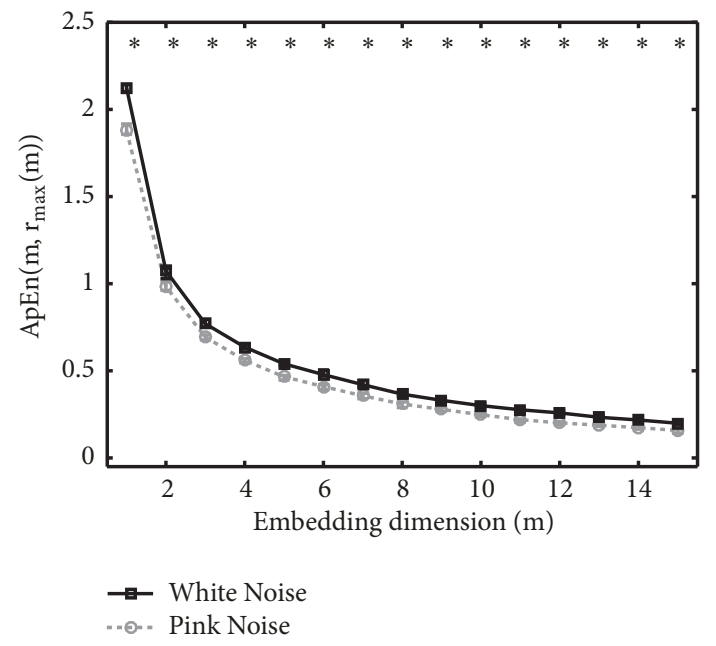

(b)

FIGURE 4: ApEn computed across embedding dimensions for pink noise (grey) and white noise (black) using different thresholds (a) $\mathrm{r}(\mathrm{m})=$ 0.2 and $(b) r(m)=r_{\max }(m) . *$ indicates statistically significant differences by Mann-Whitney $\mathrm{U}$ test $(p$-values $<0.05)$ between pink and white noise for a given embedding dimension. Data are shown as median and interquartile range over 30 realizations.

TABLE 1: $P$-values from Mann-Whitney U test comparing the index $M A p E n_{\max }$ over different synthetic time series when varying the number $\mathrm{N}$ of simulated stochastic processes.

\begin{tabular}{lcccccc}
\hline MApEn & $\mathrm{N}=5$ & $\mathrm{~N}=10$ & $\mathrm{~N}=15$ & $\mathrm{~N}=20$ & $\mathrm{~N}=25$ & $\mathrm{~N}=30$ \\
\hline $\operatorname{MIX}(0)$ vs. $\operatorname{MIX}(0.25)$ & 0.0079 & $6.4 \mathrm{e}-05$ & $6.9 \mathrm{e}-07$ & $8.0 \mathrm{e}-09$ & $9.7 \mathrm{e}-11$ & $1.2 \mathrm{e}-12$ \\
$\operatorname{MIX}(0.25)$ vs. $\operatorname{MIX}(0.5)$ & 0.0079 & $1.8 \mathrm{e}-04$ & $3.4 \mathrm{e}-06$ & $6.8 \mathrm{e}-08$ & $1.4 \mathrm{e}-09$ & $3.02 \mathrm{e}-11$ \\
$\operatorname{MIX}(0.5)$ vs. $\operatorname{MIX}(0.75)$ & 0.0079 & 0.001 & $4.02 \mathrm{e}-05$ & $1.2 \mathrm{e}-06$ & $4.9 \mathrm{e}-08$ \\
$\operatorname{MIX}(0.75)$ vs. $\operatorname{MIX}(1)$ & 0.55 & 0.43 & 0.80 & 0.32 & $4.9 \mathrm{e}-09$ \\
Pink vs. White noise & 0.0079 & $1.8 \mathrm{e}-04$ & $3.4 \mathrm{e}-06$ & $6.8 \mathrm{e}-08$ & 0.17 & $1.4 \mathrm{e}-09$ \\
\hline
\end{tabular}




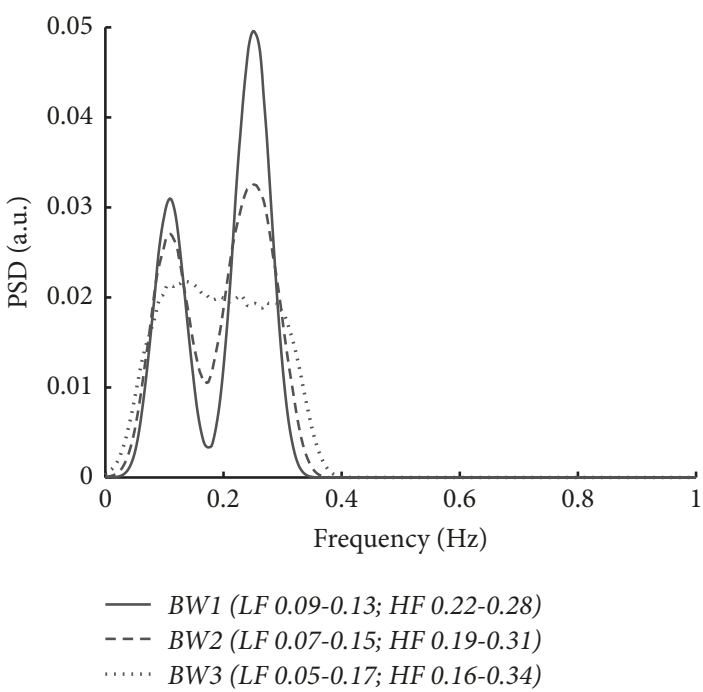

(a)

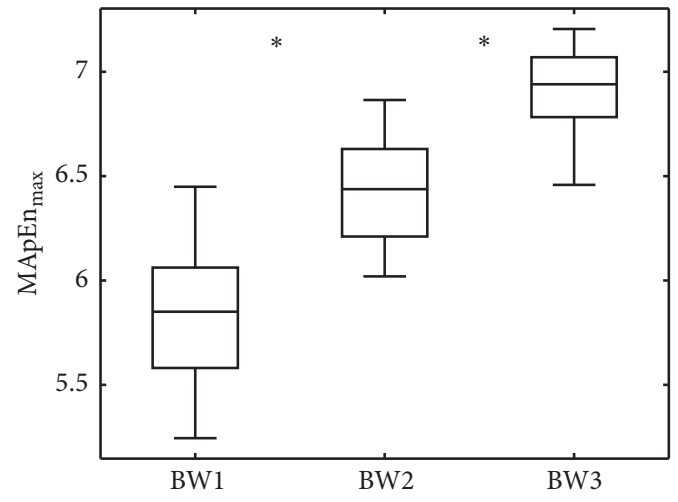

(b)

FIgURE 5: (a) Median spectrum and (b) MApEn $n_{\max }$ values obtained per type of filtered white noise time series. * indicates statistically significant differences by Mann-Whitney U test $(p$-values $<0.05)$. Data are shown as median and interquartile range over 30 realizations.

series were found only for $M A p E n_{\max }$ by Mann-Whitney $\mathrm{U}$ test (Figure 5(b)). Additionally, the dependence of the statistical significance on the number of realizations was studied for RMSSD, $P_{H F}, P_{L F}, P_{L F n}$, and $M A p E n_{\text {max }}$ (see Table S6 in Supplementary Materials).

3.2. Real Data. One subject from the young cohort and another one from the elderly cohort were discarded for analysis, as their RR time series were not suitable for ectopic beats correction due to the fact that their variability values reached the maximum allowed $H R$ variation, thus precluding distinction between normal and ectopic beats [43]

$A p E n(2,0.2), A p E n\left(2, r_{\text {max }}(2)\right), \operatorname{MApEn}(0.2), M A p E n_{\text {max }}$, $\operatorname{SampEn}(2,0.2)$, and $D_{2}$ values showed a trend for higher values in young than in elderly subjects, but only $M A p E n_{\max }$ showed statistically significantly higher values in young subjects (see Figures 6 and 7). On the other hand, all analyzed nonlinear HRV indices, with the exception of $\operatorname{MApEn}(0.2)$, were statistically significantly larger in $\mathrm{CHF}$ patients than in healthy subjects (Figures 6 and 7).

Figure 8 presents $A p E n(m, 0.2)$ and $A p E n\left(m, r_{\text {max }}(m)\right)$, as contributors to $M A p E n(0.2)$ and $M A p E n_{\max }$ (Figures 6(a) and $6(\mathrm{~b}))$ separately for each of the analyzed cohorts. The index $A p E n(m, 0.2)$ was found to have higher values in young subjects than in elderly subjects for low embedding dimensions $(m<=3)$, even if not achieving statistical significance in all cases (Figure 8(a)). Similarly, CHF patients showed higher $\operatorname{ApEn}(m, 0.2)$ values than healthy subjects for low values of the embedding dimension $m$ (Figure $8(\mathrm{c})$ ). For large embedding dimensions, opposite results were found in the two comparisons. Similar results were found for $\operatorname{ApEn}(m, 0.1)$ and $A p E n(m, 0.15)$ (see Figure S1 in Supplementary Materials, where additional results are presented for other commonly used $r$ values). Regarding $A p E n\left(m, r_{\max }(m)\right)$, statistically significantly higher values were found in young versus elderly and in CHF patients versus healthy subjects for most values of the embedding dimension $m$, while for the remaining values of $m$ the compared values could not be statistically distinguished.

HRM and RMSSD values are displayed in Figure 9 for the studied groups. No statistically significant differences were found for HRM when comparing young and elderly subjects, although a tendency to lower $H R M$ values in the elderly group was observed. HRM was statistically significantly higher in CHF patients as compared to healthy subjects. On the other hand, the RMSSD index showed statistically significant differences between both pairs of groups, with elderly subjects and CHF patients presenting reduced RMSSD values.

Values of the frequency-domain HRV indices $P_{L F}, P_{H F}$, and $P_{L F n}$ are shown in Figure 10 for the four studied groups. Significant differences were found for $P_{L F}$ and $P_{H F}$ in the two comparisons. $P_{L F n}$ only showed statistically significant differences for the comparison between CHF patients and healthy subjects.

Figure 11 illustrates the relationship between $M A p E n_{\max }$ and RMSSD (a) and $P_{H F}$ (b). Correlation coefficients computed over the four analyzed groups are shown in Table 2.

\section{Discussion}

4.1. Searching for Standardizing Approximate Entropy. Nonlinear HRV analysis has extended the description of ANS regulation of cardiac electrical activity. The use of nonlinear HRV indices has provided new physiological insights into ventricular dysfunction, ventricular tachycardia, obstetrical complications under anesthesia, mental disorders, and aging, among others [3-9]. Nevertheless, the physiological interpretation of some common nonlinear HRV indices could be biased by the a priori selection of parameter values intrinsic 


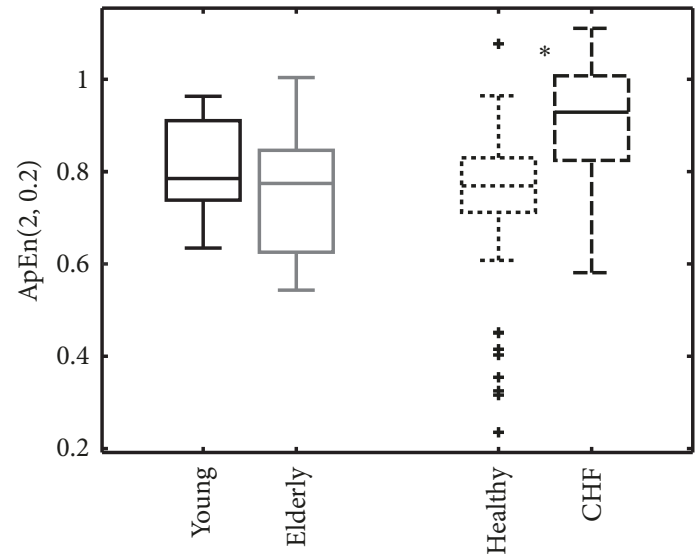

(a)

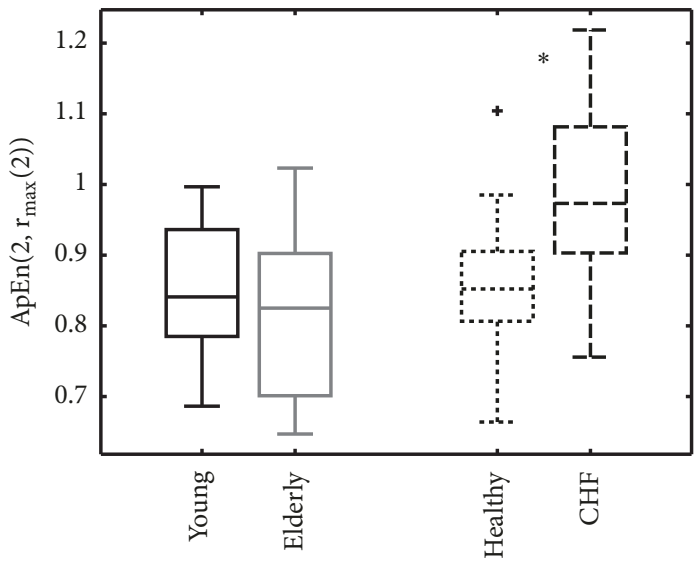

(c)

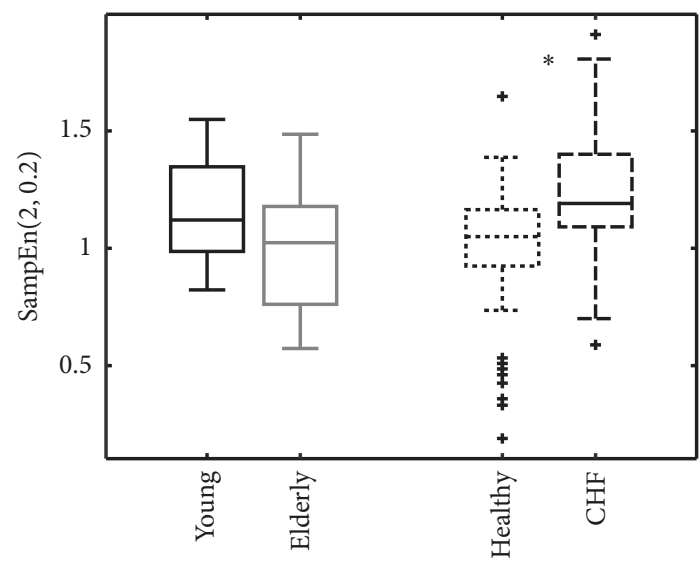

(b)

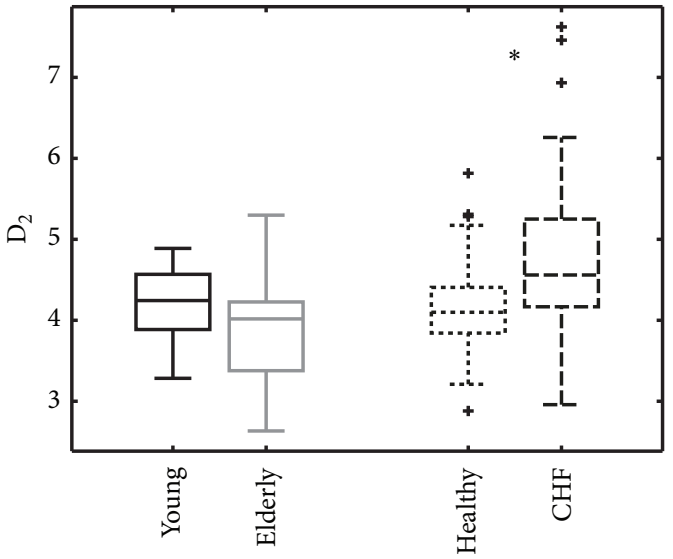

(d)

Figure 6: Regularity and complexity indices: (a) $\operatorname{ApEn}(2,0.2)$; (b) $\operatorname{SampEn}(2,0.2)$; (c) $A p E n\left(2, r_{\max }(2)\right.$ ); and (d) $D_{2}$ comparing young versus elderly, and healthy subjects versus CHF patients. * indicates statistically significant differences by Mann-Whitney U test ( $p$-values $<0.05$ ) between groups. Data are shown as median and interquartile range.

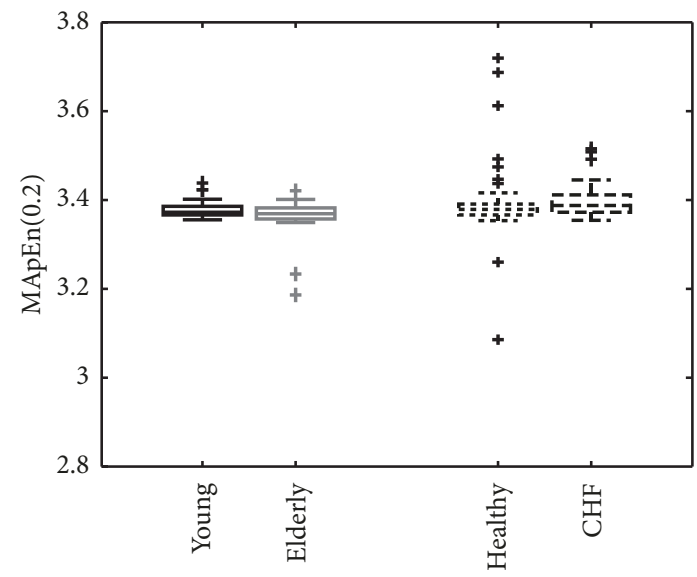

(a)

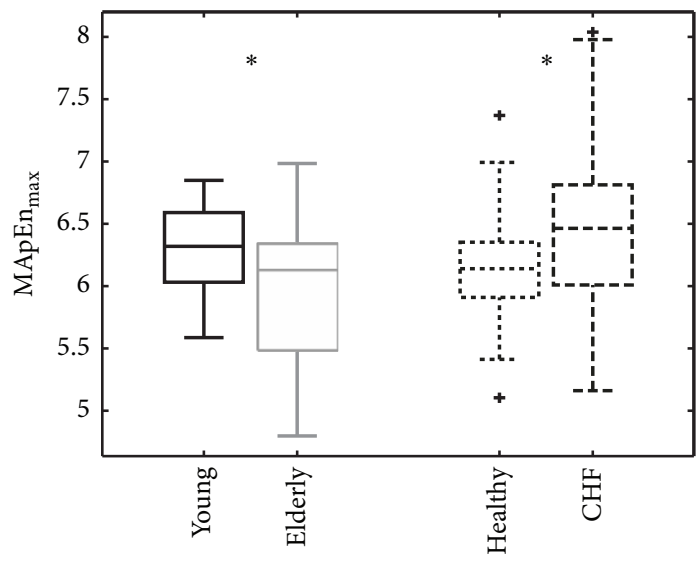

(b)

Figure 7: (a) $M A p E n(0.2)$ and (b) $M A p E n_{\max }$ values computed for young (solid black) versus elderly (solid grey), and healthy subjects (dotted) versus CHF patients (dashed). * indicates statistically significant differences by Mann-Whitney $\mathrm{U}$ test $(p$-values $<0.05)$ between groups. Data are shown as median and interquartile range. 


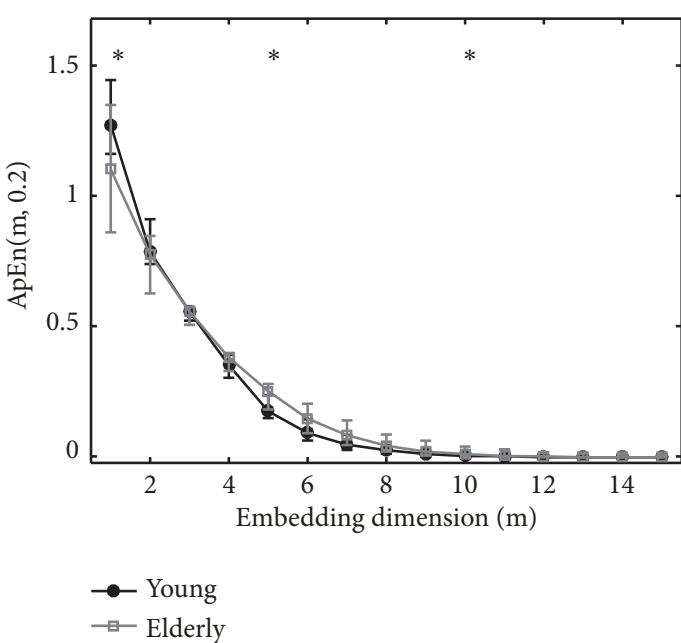

(a)

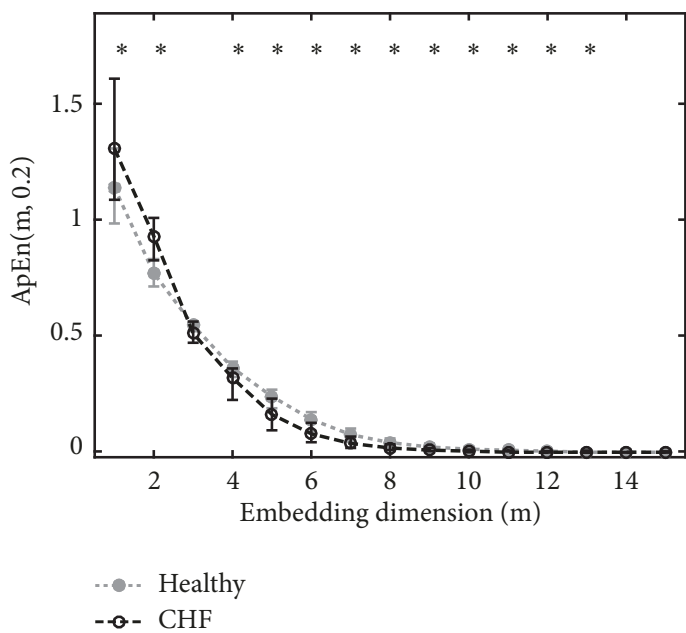

(c)

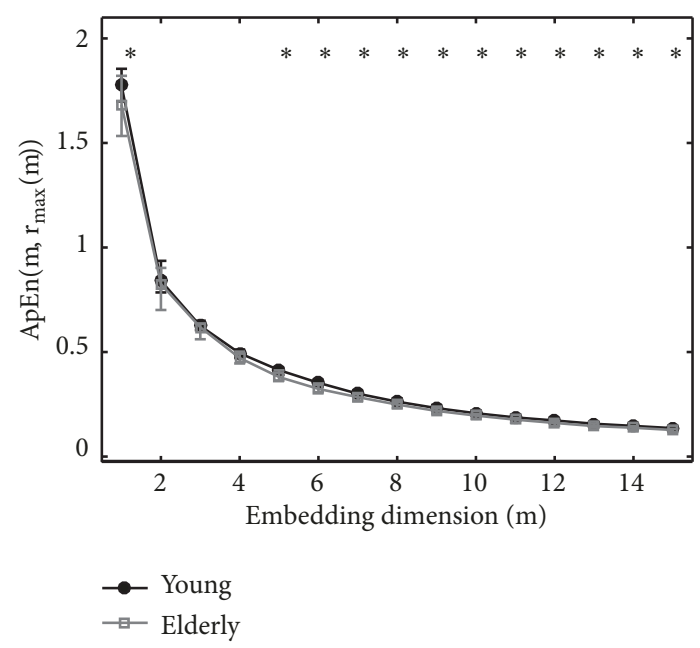

(b)

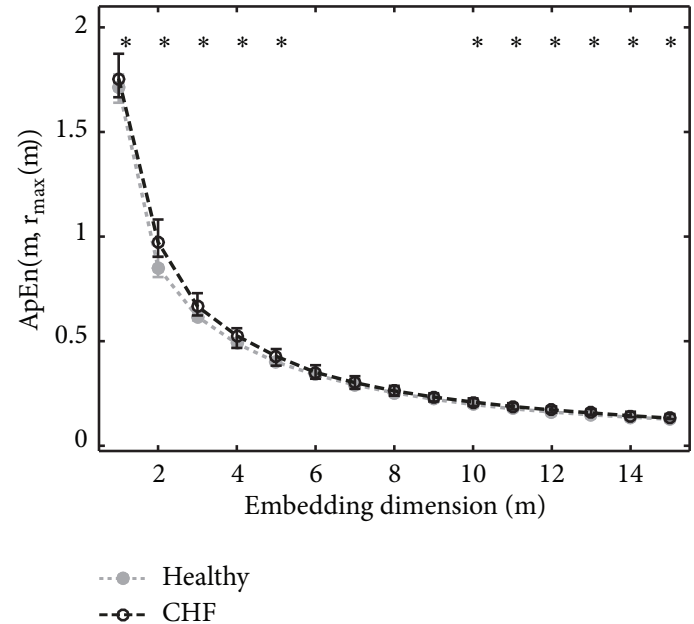

(d)

FiguRE 8: Approximate entropy values computed for thresholds set at 0.2 and $r_{\max }(\mathrm{m})$ across embedding dimensions in young (black) and elderly (grey) groups shown in (a) and (b), and in healthy subjects (dotted grey) and CHF patients (dashed black) during the night period in (c) and $(\mathrm{d}) . *$ indicates statistically significant differences by Mann-Whitney $\mathrm{U}$ test $(p$-values $<0.05)$ between groups for a given embedding dimension. Data are shown as median and interquartile range.

TABLE 2: Spearman correlation coefficients between $M A p E n_{\max }$ and RMSSD and between $M A P E n_{\max }$ and $P_{H F}$.

\begin{tabular}{|c|c|c|c|c|}
\hline & \multicolumn{2}{|c|}{$M A p E n_{\max }$ vs. $R M S S D$} & \multicolumn{2}{|c|}{$M A p E n_{\max }$ vs. $P_{H F}$} \\
\hline & $\rho$ & $p$-value & $\rho$ & $p$-value \\
\hline Young & 0.02 & 0.93 & 0.21 & 0.37 \\
\hline Elderly & -0.04 & 0.86 & 0.08 & 0.73 \\
\hline Healthy & -0.13 & 0.26 & 0.023 & 0.85 \\
\hline $\mathrm{CHF}$ & -0.57 & 0.0001 & -0.65 & 0.0001 \\
\hline
\end{tabular}

to their definitions. Some investigations have addressed the automatic search for those parameter values based on specific time series characteristics [32, 35, 38]. Indices like TotalApEn and maximum approximate entropy are still dependent on the definition of the embedding dimension $m$. The values of $m$ vary from one study to another even though there is a general tendency to set $m=2$ to estimate $\operatorname{ApEn}(2$, $\left.r_{\max }(2)\right)$. In this study, the index $M A p E n_{\text {max }}$ has been proposed based on summing the maximal approximate entropy values over all analyzed embedding dimensions. Although the computation of $M A p E n_{\text {max }}$ requires setting a maximum embedding dimension value, the relative contribution of $A p E n\left(m, r_{\max }(m)\right)$ values to the final $M A p E n_{\text {max }}$ estimation largely decreases as $m$ increases, thus rendering $M A p E n_{\max }$ 


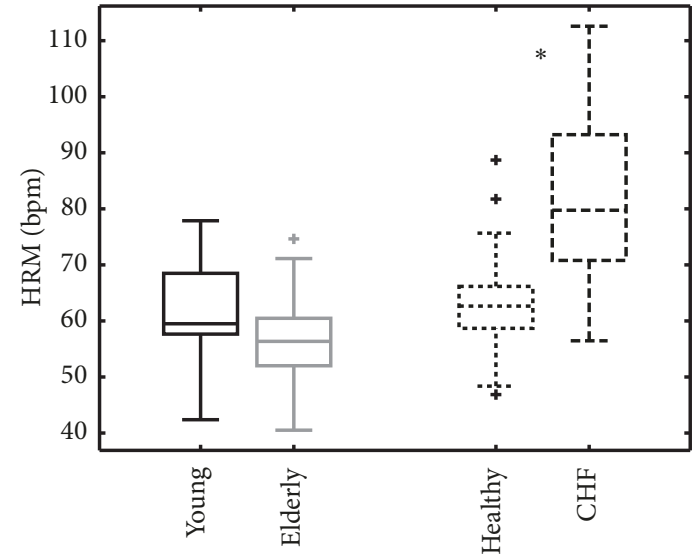

(a)

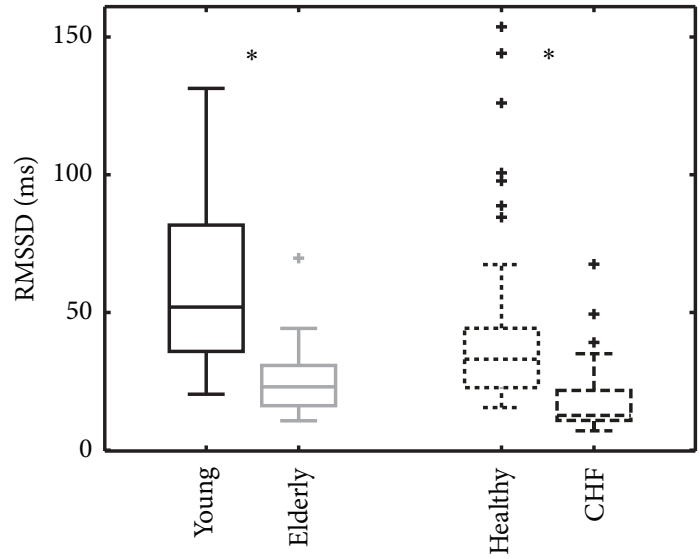

(b)

Figure 9: (a) HRM and (b) RMSSD values in young versus elderly and CHF patients versus healthy subjects during the night period. * indicates statistically significant differences by Mann-Whitney $U$ test $(p$-values $<0.05$ ) between groups.

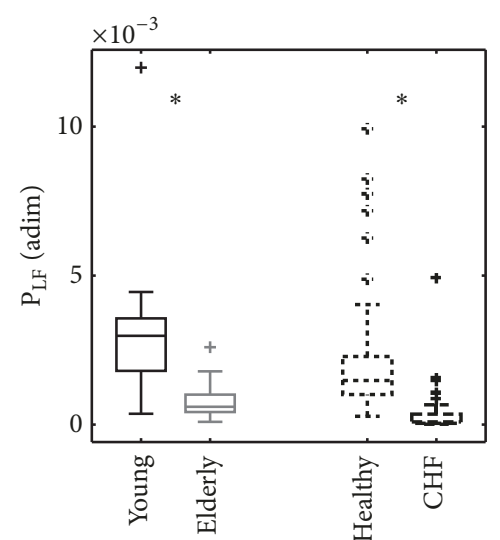

(a)

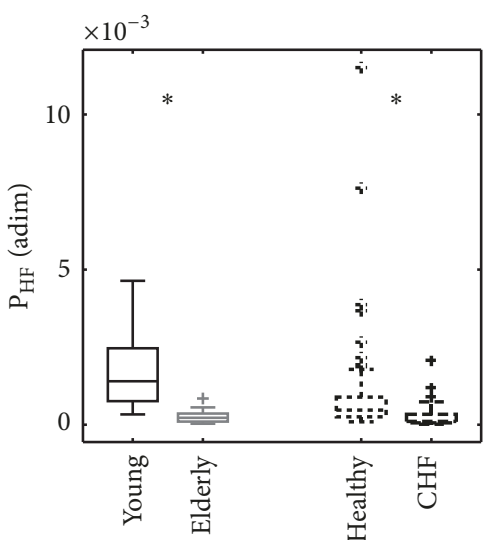

(b)

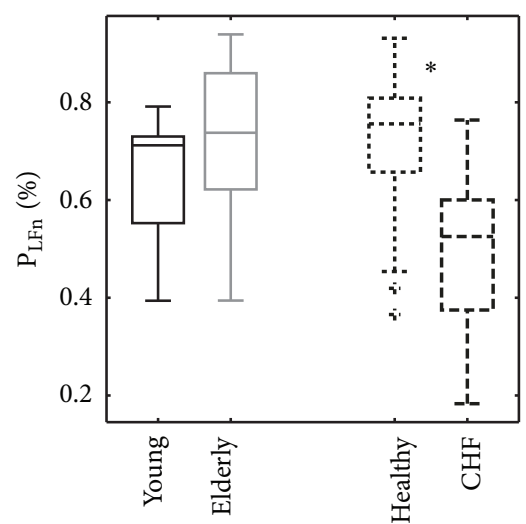

(c)

Figure 10: Frequency-domain indices: (a) $P_{L F}$; (b) $P_{H F}$; and (c) $P_{L F n}$ computed comparing young and elderly, and CHF patients and healthy subjects during the night period. * indicates statistically significant differences by Mann-Whitney $\mathrm{U}$ test $(p$-values $<0.05)$ between groups.

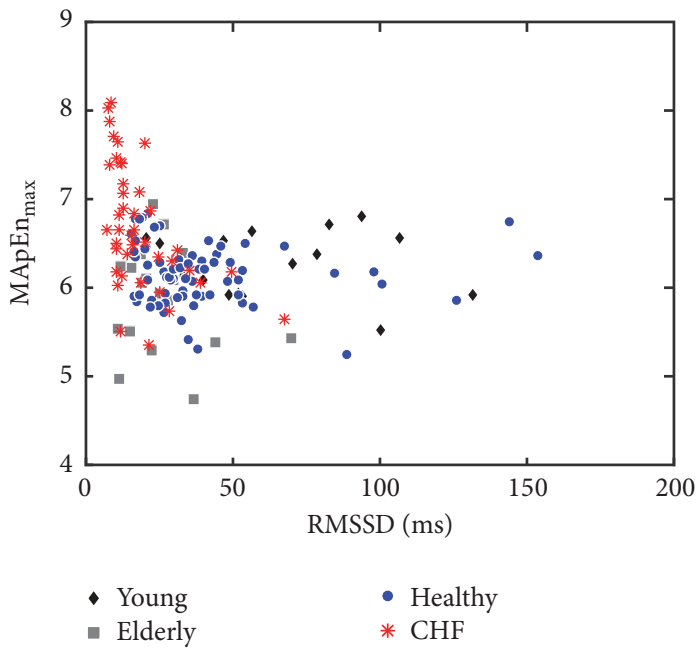

(a)

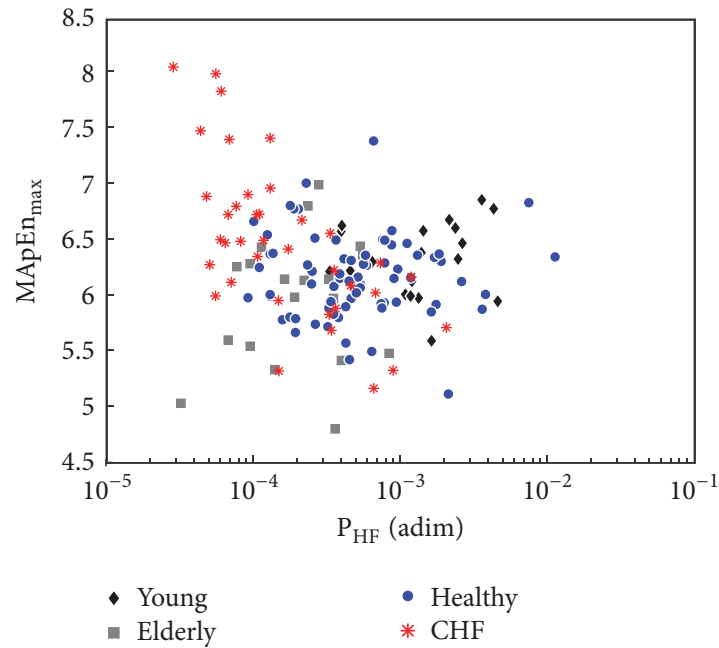

(b)

FIGURE 11: Scatter plots showing the relationship between $M A p E n_{\max }$ and $R M S S D$ (a) and between $M A p E n_{\max }$ and $P_{H F}$ (b). 
practically independent of such selection. In fact, in this study $A p E n\left(m, r_{\max }(m)\right)$ was found to present a remarkable decay for $m$ varying from 1 to less than 10 , while it remained approximately constant or with a very slow decay from that point onwards. Thus, a value of 15 or any other larger value for the maximum embedding dimension is expected to provide essentially the same outcomes. As a consequence, the proposed index, $M A p E n_{\max }$, can be seen as an entropybased index not requiring a priori parameter definition for its computation. The algorithm described in [45] for $D_{2}$ estimation was used in this study to compute the analyzed nonlinear indices. By using this algorithm, nonlinear HRV indices could be computed in less than a second for a 300sample time series, whereas the computational time for the sequential algorithm over the same time series was 1086 seconds (18 mins), in both cases using a Windows ${ }^{\circledR} 7$ based PC, Intel ${ }^{\circledR}$ Core $^{\mathrm{TM}}$ i7 $3.5 \mathrm{GHz}, 16 \mathrm{~Gb}$ RAM with Matlab $^{\circledR}$ R2015a.

Other SampEn-based indices measuring time series irregularity such as rank entropy, permutation entropy, and bubble entropy have been proposed as methods aimed at providing entropy definitions independent of a priori selected parameter values $[25,27,29]$. The multiscale extension of these SampEn-based methods, by considering the coarse-graining derived series, served as a basis for this study to formulate a multidimensional approach, overcoming the coarse-graining limitations for short-term HRV data analysis.

4.2. Analysis of Synthetic Data. The proposed index $M A p E n_{\max }$ has been tested for characterization of synthetic time series. Statistically significantly larger values of $M A p E n_{\max }$ were found for white than for pink noise. This behavior was also reproduced by all other tested nonlinear HRV indices including $\operatorname{MApEn}(0.2)$, although such differences were found to be attenuated when using a fixed tolerance threshold as compared to using the one leading to maximum entropy.

The evaluation of the proposed marker over synthetic data generated by $\operatorname{MIX}(P)$ processes revealed that the greater the stochastic level, the higher the value of $M A p E n_{\text {max }}$, which is in agreement with the results shown by other nonlinear HRV indices, like $D_{2}, A p E n$, and SampEn, describing complexity of the time series. On the contrary, the use of a fixed threshold $r$, e.g., with value 0.2 , in the computation of $\operatorname{MApEn}(r)$ was not able to reflect the level of randomness of the $\operatorname{MIX}(P)$ time series.

An additional simulation study was carried out by analyzing three types of filtered white noise time series whose timeand frequency-domain indices were statistically similar. The analysis showed that $M A p E n_{\max }$ provides complementary information to that from time- and frequency-domain indices. According to the simulation results, $M A p E n_{\max }$ was sensitive to changes in spectrum shape involving changes in HRV regularity. The narrower the spectral components, the lower the $M A P E n_{\max }$ value is, according to a more regular signal.

In the comparisons of simulated populations, the number of stochastic processes is expected to influence the statistical significance. Results have been presented to confirm the decrease in the $p$-values when the proposed index $M A p E n_{\max }$ was compared over different synthetic populations with an increasingly higher number of simulated processes. The number of processes required to attain a given significance was derived for each performed statistical comparison. Our results, in line with previous studies published in the literature [48], point out the importance of reporting the number of considered processes along with the $p$-value of any statistical comparison over synthetically generated data.

4.3. Aging and Congestive Heart Failure Assessment by Nonlinear HRV Analysis. The effect of aging was evaluated in healthy subjects during supine resting conditions, with subjects being awake. Lower values were found in the elderly cohort with respect to the young one for all analyzed nonlinear indices, with differences being statistically significant for our proposed $M A p E n_{\text {max }}$ index. Our results are in agreement with previous studies where aging has been reported as a cause for decreased complexity and irregularity of the beatto-beat RR series $[8,20,38,41,49]$. This reduction has been associated with an impairment to adapt against external or internal perturbations $[41,50]$. HRM, RMSSD, and $P_{H F}$ values were also decreased, while $P_{L F n}$ values were increased in the elderly cohort, pointing to a potential enhancement in the sympathetic modulation of sinoatrial node activity.

The impact of CHF was assessed by comparing all our analyzed indices between failing patients and healthy controls during the night period while they were sleeping. According to the results obtained for all the studied nonlinear HRV indices, including the proposed $M A p E n_{\max }$ index, statistically significantly higher complexity and irregularity were found in CHF patients with respect to healthy subjects. Similar results were found when restricting the age of the individuals to the range covering from 55 to 75 years old, pointing out that age has no relevant effect on the comparison between CHF patients and healthy subjects for the compared cohorts. Similarly, NYHA class had no impact on the distinction between CHF patients and healthy subjects, as demonstrated by restricting the analysis to $\mathrm{CHF}$ patients in NYHA classes III-IV, which led to similar results to those found for the whole CHF population (see Figure S2 in Supplementary Materials for additional details on the performed comparisons).

Spectral HRV analysis showed a significant decrease in $P_{L F n}$ for CHF patients with respect to healthy subjects. In previous studies, CHF has been reported to be associated with an increase in the sympathetic tone and a decreased peripheral response to adrenergic input [51, 52]. Studies considering pharmacological blockades have demonstrated that sympathetic blockade leads to increases in $A p E n$ and decreases in $P_{L F}$ and $P_{L F n}$, while sympathetic activation decreases the values of all nonlinear indices and increases $P_{L F n}[53,54]$. Thus, an increase in $P_{L F n}$ as well as a decrease in irregularity/complexity indices could be expected in CHF patients when compared to healthy subjects. However, in our data both a decrease in $P_{L F n}$ and an increase in irregularity/complexity indices were observed in CHF with respect 
to controls. A decrease in the low frequency content of HRV has been related to the progression of heart failure in CHF patients with advanced disease [52]. This apparently contradictory results may be explained by a decreased responsiveness to sympathetic modulation [55].

CHF has been characterized by ApEn in the literature, but results have been found to be dependent on the applied method [56]. For instance, the use of a pointprocess paradigm for describing the heartbeat generation led to greater irregularity and/or complexity values in healthy subjects as compared to CHF patients, whereas the use of raw RR interval time series showed the opposite tendency [56]. MSE, which extends the classical SampEn definition to a time-scale representation, has been applied to describe heart rate dynamics in CHF [20]. Costa et al. reported that healthy subjects presented greater MSE values than CHF patients, but in particular the elderly healthy cohort showed lower MSE values in the first scale than the CHF group. Although a relationship between MSE-derived indices and sympathetic and parasympathetic modulation has been reported [57], the methodological differences comparing MSE-derived indices and $M A p E n_{\text {max }}$ do not allow translation of the physiological interpretation to the present study.

In our study CHF patients and healthy subjects showed diverse $A p E n$ values, being greater in one or the other group depending on the tolerance $r$ and the embedding dimension $m$ values. Therefore, the different results obtained in the present study and in [21] could be due to the method itself (ApEn here and SampEn in [21]) and/or to the values used for the tolerance threshold $\left(r_{\max }(m)\right.$ in this study and fixed thresholds in [21]). ApEn $\left(m, r_{\max }(m)\right)$ values were found to be larger in CHF patients than in healthy subjects for all considered embedding dimensions, contrary to the lower MSE values in CHF patients reported for all scales in [21]. Additionally, to discard the effect of the segment length of analysis in underlying differences between the results presented here and results in [21], our proposed multidimensional index $M A p E n_{\max }$ was also tested for longer segments of HRV analysis (up to 4000 samples rather than 300 samples). Results confirmed that $M A p E n_{\max }$ presented essentially the same discriminative capacity between healthy and CHF groups for the different analyzed segment lengths, with higher values corresponding to CHF patients (see Figure S3 in Supplementary Materials). This led us to conclude that the selected segment length for HRV analysis was not a factor explaining the higher $M A p E n_{\max }$ values found in CHF patients.

Of note, the CHF patients included in the present study were enrolled in a long-term study to evaluate the efficacy of drugs, in particular milrinone and digoxin, and these drugs may have had an effect on autonomic modulation of heart rate. In addition, no information was available in relation to other concomitant disorders that CHF patients might suffer, such as obstructive sleep apneas, particularly considering that these were evaluated during the night period [58].

Finally, our proposed index, even if not showing remarkably higher discriminative capacity than other traditional HRV indices in separating young versus elderly subjects and CHF patients versus healthy subjects, could be capturing different information from that of those other indices and, consequently, it might prove useful as a complementary tool to characterize the effects of diseases or conditions. This is supported by the simulation study considering filtered white noise time series whose time- and frequency-domain indices were invariant. Other studies have also reported that the combination of entropy-based indices with time- and frequency-domain ones, when analyzed in patient populations similar to those of the present study (CHF patients versus healthy subjects), provided better classification results than by using only time- and frequency-domain indices [59, 60]. The added value of $M A p E n_{\max }$ in clinical and experimental applications should be the focus of future research studies.

\section{Conclusions}

A multidimensional approximate entropy index, $M A p E n_{\max }$, is proposed as a parameter-free entropy-based measurement. By considering maximum approximate entropy values across embedding dimensions, the proposed index increases the estimation robustness. This has been tested by evaluating the methodology on synthetic time series, including $\operatorname{MIX}(P)$ processes as well as pink and white noise, confirming that $M A p E n_{\max }$ consistently characterized the degree of randomness in the series, whereas ApEn computed by using fixed thresholds produces inconsistent results depending on the analyzed embedding dimension. $M A p E n_{\max }$ provided additional information in the analysis of the effects of aging. Specifically, $M A p E n_{\max }$ was found to be higher in elderly than young subjects, thus capturing heart rate complexity changes possibly reflecting loss of ANS adaptability in the elderly population. In addition, $M A p E n_{\max }$ was found to be increased in CHF patients as compared to healthy subjects during the night period, indicating greater HR complexity due to CHF. In conclusion, $M A p E n_{\max }$ can provide robust information on nonlinear HR dynamics with the advantage of being independent of preselected parameter values.

\section{Data Availability}

Synthetic data can be found in the following link: https://drive .google.com/open?id=1wCjF0dJmmXqdwn7Qx371GHXrN_ N0WyjP. The databases, Fantasia Database, Congestive Heart Failure RR Interval Database, BIDMC Congestive Heart Failure Database, and Normal Sinus Rhythm, used for nonlinear HRV analyses can be downloaded from http://www.physionet.org.

\section{Disclosure}

CIBER is a center of the Instituto de Salud Carlos III in assistance from the European Regional Development Fund. The computation was performed by the ICTS "NANBIOSIS", more specifically by the High Performance Computing Unit of the CIBER in Bioengineering, Biomaterials \& Nanomedicine (CIBER-BBN) at the University of Zaragoza. 


\section{Conflicts of Interest}

The authors declare that there are no conflicts of interest regarding the publication of this paper.

\section{Acknowledgments}

This work was funded under projects [TIN2014-53567-R] and [DPI2016-75458-R] by MINECO (Spain) and by BSICoS Group (T96) from Government of Aragón and European Social Fund (EU) and by the European Research Council (ERC) through project [ERC-2014-StG 638284].

\section{Supplementary Materials}

Supplementary Materials are provided. Three figures and five tables that can be found in separate files. Table S1. $r_{\max }(2)$ values computed on synthetic and real data. Mann-Whitney $\mathrm{U}$ test was used to evaluate statistical differences between consecutive row data. Data are shown in terms of median (1st|3rd) quartiles. Figure S1. Approximate entropy values computed for thresholds $r$ set at 0.1 and 0.15 across embedding dimensions in young (black) and elderly (grey) groups shown in the upper panels, and in healthy subjects (dotted grey) and CHF patients (dashed black) during the night period in the lower panels. * indicates statistically significant differences. Data are shown as median and interquartile range. Figure S2. Results of nonlinear indices computed on two subsets from healthy and CHF databases during night period, restricting age range covering from 55 to 75 years old and restricting CHF patients in NYHA classes III - IV. * indicates statistically significant differences. Data are shown as median and interquartile range. Figure S3. $M A p E n_{\text {max }}$ values computed for young (solid black) versus elderly (solid grey), and healthy subjects (dotted black) versus CHF patients (dashed grey) in time series of 4000 samples. * indicates statistically significant differences ( $p$-values < 0.05 (dark), $p$-values $<0.07$ (grey)). Data are shown as median and interquartile range. Table S2. $P$-values and AUC (Area Under Roc Curve) computed on synthetic data for the nonlinear indices. Mann-Whitney $U$ test was used to evaluate statistical differences. Table S3. $P$-values computed in the comparison between $\operatorname{ApEn}(m, 0.2)$ and $\operatorname{ApEn}\left(m, r_{\text {max }}(m)\right)$ across embedding dimensions. Mann-Whitney $U$ test was used to evaluate statistical differences. Data are shown in terms of median (IQR). Table S4. $P$-values and AUC (Area Under Roc Curve) computed on HRV data for nonlinear indices. Mann-Whitney U test was used to evaluate statistical differences. Table S5. $P$-values and AUC (Area Under Roc Curve) computed on HRV data for temporal and spectral indices. Mann-Whitney U test was used to evaluate statistical differences. Figure S4. (a) RMSSD, (b) $P_{H F}$, (c) $P_{L F}$, and (d) $P_{L F n}$ values obtained per type of filtered white noise time series. * indicates statistically significant differences by Mann-Whitney U test $(p$-values $<0.05)$. Data are shown as median and interquartile range over 30 realizations. Table S6. $P$-values from Mann-Whitney $U$ test comparing the indices RMSSD, $P_{H F}, P_{L F}, P_{L F n}$, and $M A p E n_{\max }$ over filtered white noise time series by considering BW1, BW2, and BW3, varying the number $\mathrm{N}$ of simulated processes. $P$-values $<$ 0.05 , marked in bold, are considered statistically significant. (Supplementary Materials)

\section{References}

[1] S. M. Pincus, I. M. Gladstone, and R. A. Ehrenkranz, "A regularity statistic for medical data analysis," Journal of Clinical Monitoring and Computing, vol. 7, no. 4, pp. 335-345, 1991.

[2] J. S. Richman and J. R. Moorman, "Physiological time-series analysis using approximate entropy and sample entropy," American Journal of Physiology-Heart and Circulatory Physiology, vol. 278, no. 6, pp. H2039-H2049, 2000.

[3] L. A. Fleisher, S. M. Pincus, and S. H. Rosenbaum, "Approximate entropy of heart rate as a correlate of postoperative ventricular dysfunction," Anesthesiology, vol. 78, no. 4, pp. 683-692, 1993.

[4] D. Landry P, F. M. Bennett, and N. E. Oriol, "Analysis of Heart Rate Dynamics as a Measure of Autonomic Tone in Obstetrical Patients Undergoing Epidural or Spinal Anesthesia," Regional Anesthesia, vol. 19, no. 3, pp. 189-95, 1994.

[5] A. L. Goldberger, J. E. Mietus, D. R. Rigney, M. L. Wood, and S. M. Fortney, "Effects of head-down bed rest on complex heart rate variability: Response to LBNP testing," Journal of Applied Physiology, vol. 77, no. 6, pp. 2863-2869, 1994.

[6] S. A. Schuckers, "Use of approximate entropy measurements to classify ventricular tachycardia and fibrillation," Journal of Electrocardiology, vol. 31, pp. 101-105, 1998.

[7] V. Yeragani K, R. Nadella, B. Hinze, S. Yeragani, and V. C. Jampala, "Nonlinear Measures of Heart Period Variability: Decreased Measures of Symbolic Dynamics in Patients with Panic Disorder," Depression and Anxiety, vol. 12, no. 2, pp. 67-77, 2000.

[8] F. Beckers, B. Verheyden, and A. E. Aubert, "Aging and nonlinear heart rate control in a healthy population," American Journal of Physiology-Heart and Circulatory Physiology, vol. 290, no. 6, pp. H2560-H2570, 2006.

[9] M. Sabeti, S. Katebi, and R. Boostani, "Entropy and complexity measures for EEG signal classification of schizophrenic and control participants," Artificial Intelligence in Medicine, vol. 47, no. 3, pp. 263-274, 2009.

[10] S. M. Pincus and A. L. Goldberger, "Physiological time-series analysis: what does regularity quantify?" American Journal of Physiology-Heart and Circulatory Physiology, vol. 266, no. 4, pp. H1643-H1656, 1994.

[11] V. Srinivasan, C. Eswaran, and N. Sriraam, "Approximate entropy-based epileptic EEG detection using artificial neural networks," IEEE Transactions on Information Technology in Biomedicine, vol. 11, no. 3, pp. 288-295, 2007.

[12] Q. Yuan, W. Zhou, S. Li, and D. Cai, "Epileptic EEG classification based on extreme learning machine and nonlinear features," Epilepsy Research, vol. 96, no. 1-2, pp. 29-38, 2011.

[13] D. Abásolo, R. Hornero, P. Espino, J. Poza, C. I. Sánchez, and R. De La Rosa, "Analysis of regularity in the EEG background activity of Alzheimer's disease patients with Approximate Entropy," Clinical Neurophysiology, vol. 116, no. 8, pp. 1826-1834, 2005.

[14] M. Ferrario, M. G. Signorini, G. Magenes, and S. Cerutti, "Comparison of entropy-based regularity estimators: Application to the fetal heart rate signal for the identification of fetal distress," IEEE Transactions on Biomedical Engineering, vol. 53, no. 1, pp. 119-125, 2006. 
[15] M. Costa, A. L. Goldberger, and C.-K. Peng, "Multiscale entropy analysis of complex physiologic time series," Physical Review Letters, vol. 89, no. 6, Article ID 068102, 2002.

[16] S. Pincus, "Approximate entropy (ApEn) as a complexity measure," Chaos: An Interdisciplinary Journal of Nonlinear Science, vol. 5, no. 1, pp. 110-117, 1995.

[17] M. G. Signorini, M. Ferrario, S. Cerutti, and G. Magenes, "Advances in monitoring cardiovascular signals. Contribution of nonlinear signal processing," in Proceedings of the 2011 33rd Annual International Conference of the IEEE Engineering in Medicine and Biology Society, pp. 6568-6571, Boston, MA, August 2011.

[18] V. Bari, J. F. Valencia, M. Vallverdu et al., "Refined multiscale entropy analysis of heart period and QT interval variabilities in long QT syndrome type-1 patients," in Proceedings of the 2013 35th Annual International Conference of the IEEE Engineering in Medicine and Biology Society (EMBC), pp. 5554-5557, Osaka, July 2013.

[19] F. Aletti, M. Ferrario, T. B. A. de Jesus et al., "Heart rate variability in children with cyanotic and acyanotic congenital heart disease: analysis by spectral and non linear indices." Conference proceedings: IEEE Engineering in Medicine and Biology Society, pp. 4189-4192, 2012.

[20] M. Costa, A. L. Goldberger, and C. Peng, "Multiscale entropy analysis of biological signals," Physical Review E: Statistical, Nonlinear, and Soft Matter Physics, vol. 71, no. 2, Article ID 021906, 2005.

[21] J. F. Valencia, A. Porta, M. Vallverdú et al., "Refined multiscale entropy: application to 24-h holter recordings of heart period variability in healthy and aortic stenosis subjects," IEEE Transactions on Biomedical Engineering, vol. 56, no. 9, pp. 2202-2213, 2009.

[22] L. Faes, A. Porta, M. Javorka, and G. Nollo, "Efficient computation of multiscale entropy over short biomedical time series based on linear state-space models," Complexity, vol. 2017, Article ID 1768264, 13 pages, 2017.

[23] D. Cornforth, H. F. Jelinek, and M. Tarvainen, "A comparison of nonlinear measures for the detection of cardiac autonomic neuropathy from heart rate variability," Entropy, vol. 17, no. 3, pp. 1425-1440, 2015.

[24] D. J. Cornforth, M. P. Tarvainen, and H. F. Jelinek, "How to calculate Renyi entropy from heart rate variability, and why it matters for detecting cardiac autonomic neuropathy," Frontiers in Bioengineering and Biotechnology, vol. 2, 2014.

[25] C. Bandt and B. Pompe, "Permutation entropy: a natural complexity measure for time series," Physical Review Letters, vol. 88, Article ID 174102, 2002.

[26] M. Rostaghi and H. Azami, "Dispersion Entropy: A Measure for Time-Series Analysis," IEEE Signal Processing Letters, vol. 23, no. 5, pp. 610-614, 2016.

[27] L. Citi, G. Guffanti, and L. Mainardi, "Rank-based multi-scale entropy analysis of heart rate variability," in Proceedings of the 41st Computing in Cardiology Conference, CinC 2014, pp. 597600, USA, September 2014.

[28] R. Udhayakumar, C. Karmakar, and M. Palaniswami, "Understanding Irregularity Characteristics of Short-term HRV Signals using Sample Entropy Profile," IEEE Transactions on Biomedical Engineering, pp. 1-1, 2018.

[29] G. Manis, M. Aktaruzzaman, and R. Sassi, "Bubble entropy: An entropy almost free of parameters," IEEE Transactions on Biomedical Engineering, vol. 64, no. 11, pp. 2711-2718, 2017.
[30] M. B. Kennel, R. Brown, and H. D. I. Abarbanel, “Determining embedding dimension for phase-space reconstruction using a geometrical construction," Physical Review A: Atomic, Molecular and Optical Physics, vol. 45, no. 6, pp. 3403-3411, 1992.

[31] L. Cao, "Practical method for determining the minimum embedding dimension of a scalar time series," Physica D: Nonlinear Phenomena, vol. 110, no. 1-2, pp. 43-50, 1997.

[32] S. Lu, X. Chen, J. K. Kanters, I. C. Solomon, and K. H. Chon, "Automatic selection of the threshold value $\mathrm{r}$ for approximate entropy," IEEE Transactions on Biomedical Engineering, vol. 55, no. 8, article no. 8, pp. 1966-1972, 2008.

[33] P. Castiglioni and M. Di Rienzo, "How the threshold " $r$ " influences approximate entropy analysis of heart-rate variability," in Proceedings of the Computers in Cardiology 2008, CAR, pp. 561564, Bologna, Italy, September 2008.

[34] A. Singh, B. S. Saini, and D. Singh, "An Alternative Approach to Approximate Entropy Threshold Value (r) Selection: Application to Heart Rate Variability and Systolic Blood Pressure Variability under Postural Challenge," Medical Biological Engineering Computing, vol. 54, no. 5, pp. 723-32, 2016.

[35] C. Liu, C. Liu, P. Shao et al., "Comparison of different threshold values $r$ for approximate entropy: Application to investigate the heart rate variability between heart failure and healthy control groups," Physiological Measurement, vol. 32, no. 2, pp. 167-180, 2011.

[36] J. F. Restrepo, G. Schlotthauer, and M. E. Torres, "Maximum approximate entropy and $\mathrm{r}$ threshold: A new approach for regularity changes detection," Physica A: Statistical Mechanics and its Applications, vol. 409, pp. 97-109, 2014.

[37] K. Chon, C. G Scully, and S. Lu, "Approximate Entropy for All Signals," IEEE Engineering in Medicine and Biology Magazine: The Quarterly Magazine of the Engineering in Medicine \& Biology Society, vol. 28, no. 6, pp. 18-23, 2009.

[38] R. K. Udhayakumar, C. Karmakar, and M. Palaniswami, "Approximate entropy profile: a novel approach to comprehend irregularity of short-term HRV signal," Nonlinear Dynamics, vol. 88, no. 2, pp. 823-837, 2017.

[39] J. Li and P. Shang, "Financial time series analysis using TotalCApEN and Avg-CApEN with cumulative histogram matrix," Communications in Nonlinear Science and Numerical Simulation, vol. 63, pp. 239-252, 2018.

[40] S. M. Pincus, "Approximate entropy as a measure of system complexity," Proceedings of the National Acadamy of Sciences of the United States of America, vol. 88, no. 6, pp. 2297-2301, 1991.

[41] N. Iyengar, C.-K. Peng, R. Morin, A. L. Goldberger, and L. A. Lipsitz, "Age-related alterations in the fractal scaling of cardiac interbeat interval dynamics," American Journal of PhysiologyRegulatory, Integrative and Comparative Physiology, vol. 271, no. 4, pp. R1078-R1084, 1996.

[42] A. L. Goldberger, L. A. Amaral, L. Glass et al., "PhysioBank, PhysioToolkit, and PhysioNet: components of a new research resource for complex physiologic signals.," Circulation, vol. 101, no. 23, pp. E215-E220, 2000.

[43] J. Mateo and P. Laguna, "Analysis of heart rate variability in the presence of ectopic beats using the heart timing signal," IEEE Transactions on Biomedical Engineering, vol. 50, no. 3, pp. 334343, 2003.

[44] J. Bolea, E. Pueyo, M. Orini, and R. Bailón, "Influence of Heart Rate in Non-linear HRV Indices as a Sampling Rate Effect Evaluated on Supine and Standing," Frontiers in Physiology, vol. 7, 2016. 
[45] J. Bolea, P. Laguna, J. M. Remartínez, E. Rovira, A. Navarro, and R. Bailón, "Methodological Framework for Estimating the Correlation Dimension in HRV Signals," Computational and Mathematical Methods in Medicine, vol. 2014, Article ID 129248, 11 pages, 2014.

[46] R. Bailón, G. Laouini, C. Grao, M. Orini, P. Laguna, and O. Meste, "The integral pulse frequency modulation model with time-varying threshold: Application to heart rate variability analysis during exercise stress testing," IEEE Transactions on Biomedical Engineering, vol. 58, no. 3, pp. 642-652, 2011.

[47] Task Force of the ESC-NASPE, "Heart rate variability: standards of measurement, physiological interpretation and clinical use. Task Force of the European Society of Cardiology and the North American Society of Pacing and Electrophysiology," Circulation, vol. 93, no. 5, pp. 1043-1065, 1996.

[48] J. W. White, A. Rassweiler, J. F. Samhouri, A. C. Stier, and C. White, "Ecologists should not use statistical significance tests to interpret simulation model results," Oikos, vol. 123, no. 4, pp. 385-388, 2014.

[49] D. T. Kaplan, M. I. Furman, S. M. Pincus, S. M. Ryan, L. A. Lipsitz, and A. L. Goldberger, "Aging and the complexity of cardiovascular dynamics," Biophysical Journal, vol. 59, no. 4, pp. 945-949, 1991.

[50] M. Reardon and M. Malik, "Changes in heart rate variability with age," Pacing and Clinical Electrophysiology, vol. 19, no. 11, pp. 1863-1866, 1996.

[51] M. G. Kienzle, D. W. Ferguson, C. L. Birkett, G. A. Myers, W. J. Berg, and D. J. Mariano, "Clinical, hemodynamic and sympathetic neural correlates of heart rate variability in congestive heart failure," American Journal of Cardiology, vol. 69, no. 8, pp. 761-767, 1992.

[52] S. Guzzetti, C. Cogliati, M. Turiel, C. Crema, F. Lombardi, and A. Malliani, "Sympathetic predominance followed by functional denervation in the progression of chronic heart failure," European Heart Journal, vol. 16, no. 8, pp. 1100-1107, 1995.

[53] M. Osaka, H. Saitoh, H. Atarashi, and H. Hayakawa, "Correlation Dimension of Heart Rate Variability: A New Index of Human Autonomic Function," Frontiers of Medical and Biological Engineering: The International Journal of the Japan Society of Medical Electronics and Biological Engineering, vol. 5, no. 4, pp. 289-300, 1993, http://www.ncbi.nlm.nih.gov/pubmed/8136314.

[54] J. Bolea, E. Pueyo, P. Laguna, and R. Bailón, "Non-Linear HRV Indices under Autonomic Nervous System Blockade," in Proceedings of the Annual International Conference of the IEEE Engineering in Medicine and Biology Society, EMBS, pp. 32523255, August 2014.

[55] S. Guzzetti, R. Magatelli, E. Borroni, and S. Mezzetti, "Heart rate variability in chronic heart failure," Autonomic Neuroscience: Basic and Clinical, vol. 90, no. 1-2, pp. 102-105, 2001.

[56] Z. Chen, E. N. Brown, and R. Barbieri, "Characterizing nonlinear heartbeat dynamics within a point process framework," IEEE Transactions on Biomedical Engineering, vol. 57, no. 6, pp. 1335-1347, 2010.

[57] L. E. V. Silva, R. M. Lataro, J. A. Castania et al., "Multiscale entropy analysis of heart rate variability in heart failure, hypertensive, and sinoaortic-denervated rats: Classical and refined approaches," American Journal of Physiology-Regulatory, Integrative and Comparative Physiology, vol. 311, no. 1, pp. R150R156, 2016.

[58] M. T. Naughton and T. D. Bradley, "Sleep apnea in congestive heart failure," Clinics in Chest Medicine, vol. 19, no. 1, pp. 99-113, 1998.
[59] D. Luo, W. Pan, Y. Li, K. Feng, and G. Liu, "The interaction analysis between the sympathetic and parasympathetic systems in CHF by using transfer entropy method," Entropy, vol. 20, no. 10, p. 795, 2018.

[60] C. Liu and R. Gao, "Multiscale Entropy Analysis of the Differential RR Interval Time Series Signal and Its Application in Detecting Congestive Heart Failure," Entropy, vol. 19, no. 6, p. 251, 2017. 


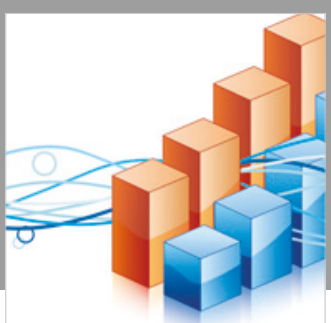

Advances in

Operations Research

\section{-n-m}
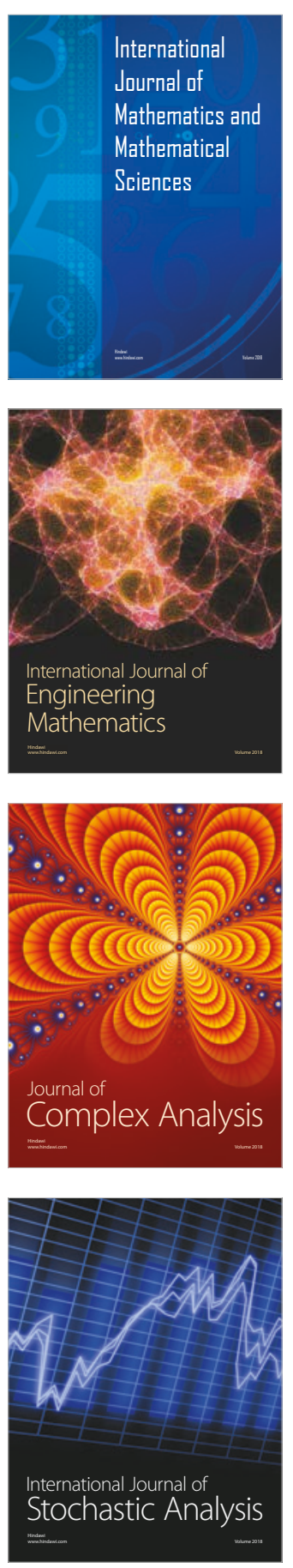
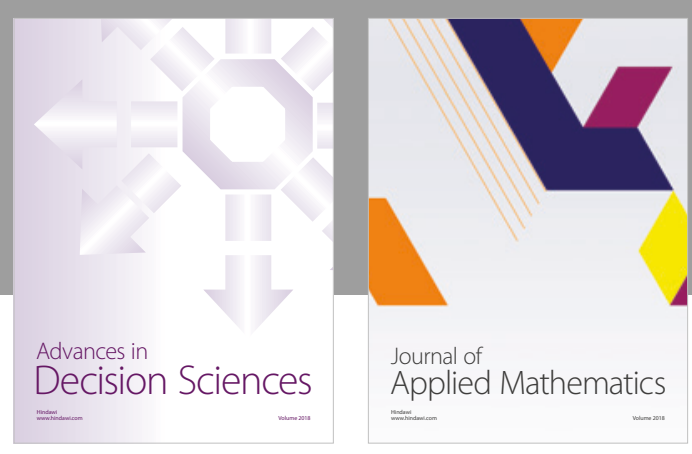

Journal of

Applied Mathematics
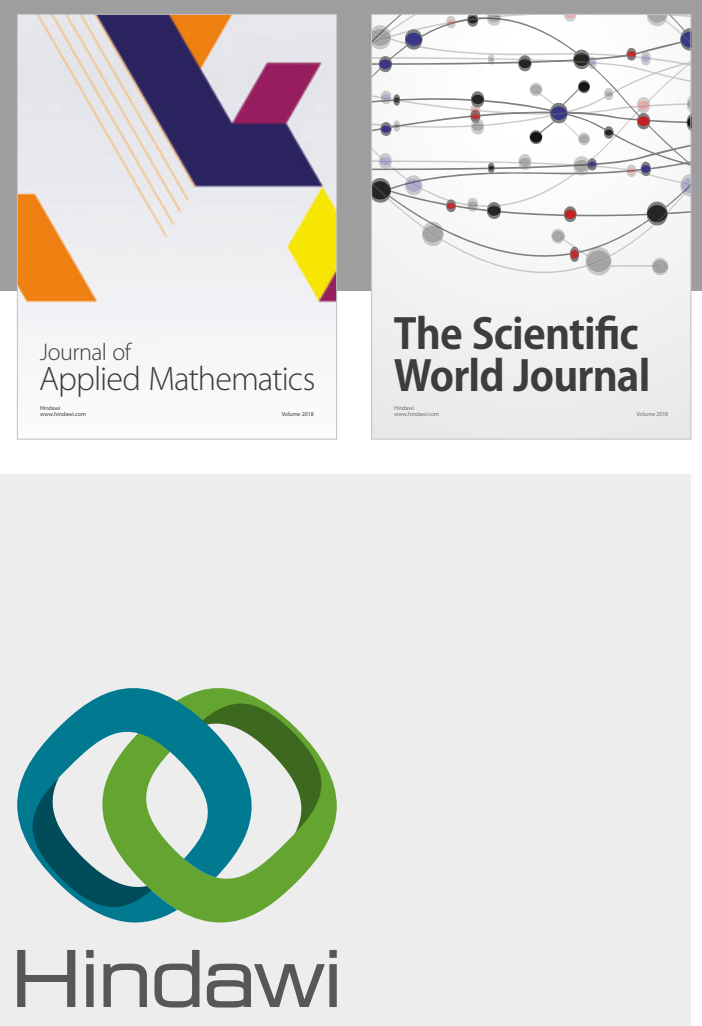

Submit your manuscripts at

www.hindawi.com

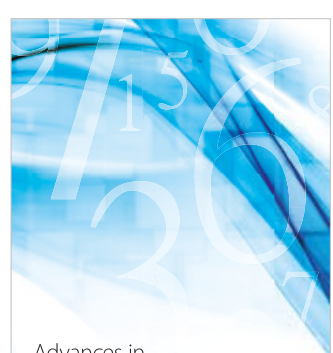

Advances in
Numerical Analysis
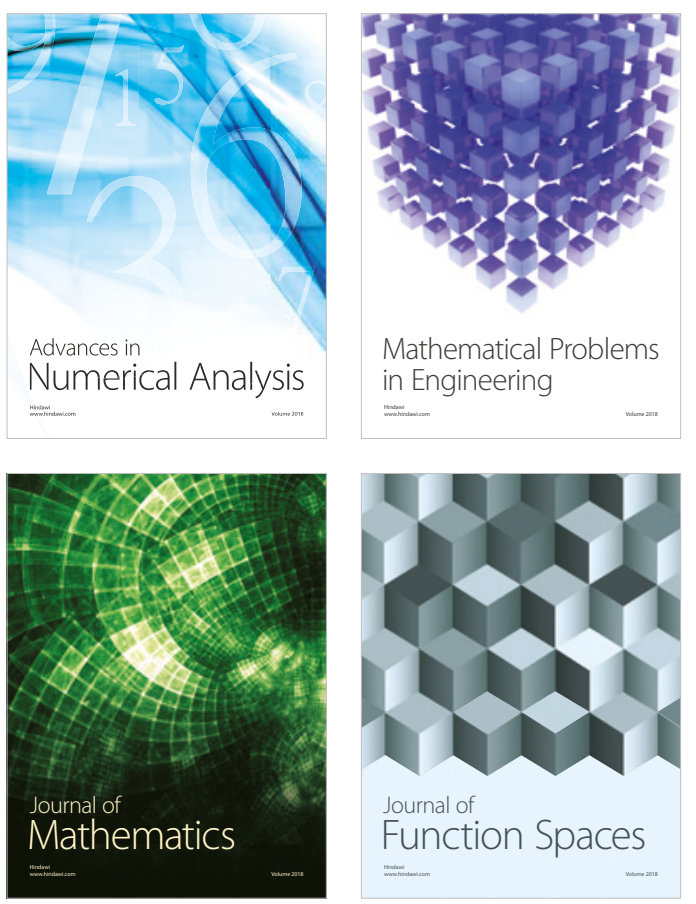

Mathematical Problems in Engineering

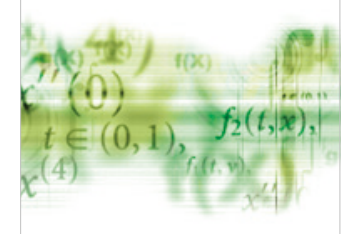

International Journal of

Differential Equations

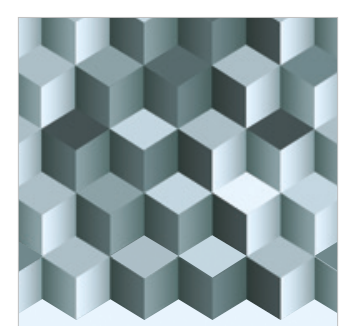

Journal of

Function Spaces
The Scientific

World Journal

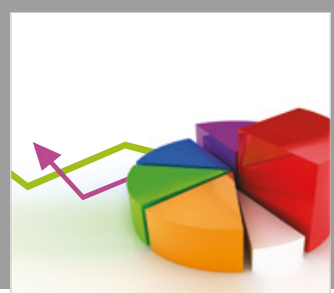

Journal of

Probability and Statistics
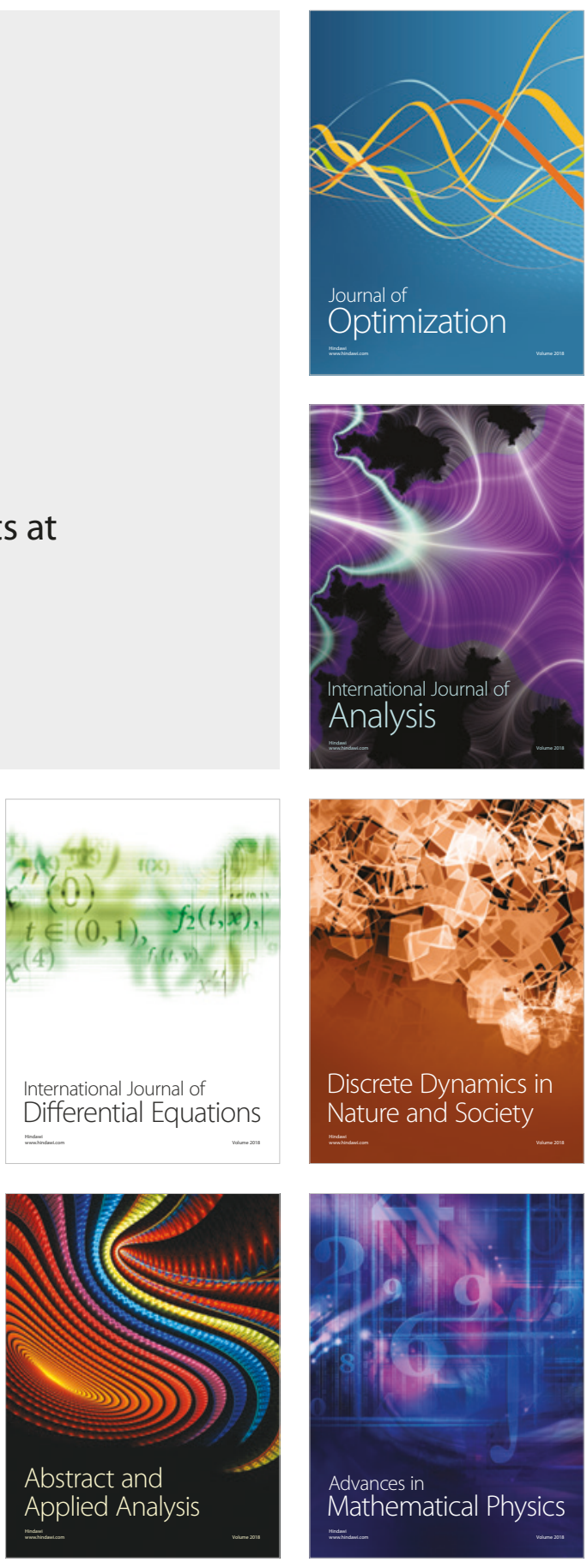